\title{
Camera Model Identification Based on DCT Coefficient Statistics
}

\author{
Thanh Hai Thai*, Florent Retraint and Rémi Cogranne** \\ ICD - LM2S - University of Technology of Troyes - UMR STMR CNRS \\ 12, rue Marie Curie - CS 42060 - 10004 Troyes cedex - France
}

(C) 2013 Elsevier B.V. All rights reserved. Accepted version provided for non-commercial research and education use. Final version published in Digital signal processing, Vol. 40, no. 5, pp. 88-100, May 2015 and is available at doi: 10.1016/j.dsp.2015.01.002. This article appeared in a journal published by Elsevier. The attached copy is furnished to the author for internal non-commercial research and education use, including for instruction at the authors institution and sharing with colleagues. Other uses, including reproduction and distribution, or selling or licensing copies, or posting to personal, institutional or third party websites are prohibited.

\begin{abstract}
The goal of this paper is to design a statistical test for the camera model identification problem from JPEG images. The approach relies on the camera fingerprint extracted in the Discrete Cosine Transform (DCT) domain based on the state-of-the-art model of DCT coefficients. The camera model identification problem is cast in the framework of hypothesis testing theory. In an ideal context where all model parameters are perfectly known, the Likelihood Ratio Test is presented and its performances are theoretically established. For a practical use, two Generalized Likelihood Ratio Tests are designed to deal with unknown model parameters such that they can meet a prescribed false alarm probability while ensuring a high detection performance. Numerical results on simulated and real JPEG images highlight the relevance of the proposed approach.
\end{abstract}

Keywords: Digital Forensics, Camera Model Identification, Hypothesis Testing, Natural Image Model, Discrete Cosine Transform.

\section{Introduction}

The evolution of digital imaging technology and information technologies in the past decades has raised a number of information security challenges. Digital images can be easily edited, altered or falsified because of a large availability of low-cost image editing tools. Consequently, the credibility and trustworthiness of digital images have been eroded. This is more crucial when falsified images that are utilized as evidence in a courtroom could mislead the judgement and lead to either imprisonment for the innocent or freedom for the guilty. Digital image forensics has

\footnotetext{
(C) 2013 Elsevier B.V. All rights reserved. Accepted version provided for non-commercial research and education use. Final version published in Digital signal processing, Vol. 40, no. 5, pp. 88-100, May 2015 and is available at doi: 10.1016/j.dsp.2015.01.002. This article appeared in a journal published by Elsevier. The attached copy is furnished to the author for internal non-commercial research and education use, including for instruction at the authors institution and sharing with colleagues. Other uses, including reproduction and distribution, or selling or licensing copies, or posting to personal, institutional or third party websites are prohibited.

* Corresponding author.

${ }^{* *}$ With the financial support from Champagne-Ardenne region, IDENT project.

Email address: thanh_hai.thai@utt.fr (Thanh Hai Thai)
} 
been emerged in response to the increasing need to verify the trustworthiness of digital images, see [1] and references therein for detailed introductions.

\subsection{State of the Art}

One of the key problems of digital image forensics is identification of image origin, which aims to verify whether a given image was acquired by a specific camera or determine camera models/brands as well as types of imaging mechanism (e.g. scanners, cell-phone cameras, or computer graphics). Basically, when an image is captured by a camera, it is stored with the metadata headers in the memory storage device. The metadata, e.g. Exchangeable Image File (EXIF) and JPEG headers, contain all recording and compression history. Therefore, a simplest way to determine the image's source is to read out directly from the metadata. However, such metadata headers are not always available in practice if the image is resaved in a different format or recompressed. Another problem is that the metadata headers are not reliable as they can be easily removed or modified using low-cost editing tools. Therefore, it is desirable for law enforcement agencies to build up a set of reliable forensic tools for image origin identification.

In general, there are two approaches to address the problem of image origin identification. Active forensics involves generating extrinsically security measures such as digital watermarks [2] and digital signatures [3], referred to as extrinsic fingerprints, and adding to the image file. However, active forensics is of limited extent due to many strict constraints in its protocols. In order to solve the problem of image origin identification in its entirety, passive forensics has been quickly evolved. In contrast to active forensics, passive forensics does not impose any constraint, nor require any prior information including the original reference image. Forensic analysts have only the suspect image at their disposal and must explore useful information from that image to gather forensic evidence and trace the acquisition device. The common philosophy in passive approach is to rely on inherent intrinsic fingerprints that the digital camera leaves in a given image. Passive forensics can be widely applied to millions of images that circulate daily on communication networks.

This paper mainly addresses the origin identification of images acquired by digital cameras based on passive approach. It is important to distinguish the problem of camera instance identification and the problem of camera model/brand identification. More specifically, fingerprints used for camera instance identification should capture individuality, especially cameras coming from the same brand and model. For camera model/brand identification, it is necessary to exploit fingerprints that are shared between cameras of the same model/brand but discriminative for different camera models/brands. Passive forensic methods proposed for those problems can be divided into two fundamental categories.

Technically, any method proposed for image origin identification must respond to following questions:

1. Which fingerprints are utilized for identification?

2. How to extract these fingerprints accurately from a given image? 
3. Under which frameworks is the method designed to exploit the discriminability of fingerprints extracted from images captured by different sources ${ }^{1}$ and to calculate the similarity of fingerprints extracted from images captured by the same source?

Every stage from real-world scene acquisition to image storage can provide intrinsic fingerprints for forensic analysis. Therefore to design a camera fingerprint, it is necessary to study the image processing pipeline of a digital camera. Although the image processing pipeline is common for most cameras, each processing step is performed according to manufacturers' own design. Thus the information left by each processing step is useful to trace down to the device source.

Several fingerprints have been proposed in the literature. Sensor Pattern Noise (SPN), which is caused by imperfections during the manufacturing process and non-uniformity of photo-electronic conversion due to inhomogeneity of silicon wafers, is used in [4] for camera instance identification. Two main components of the SPN are the Fixed Pattern Noise (FPN) and the Photo-Response Non-Uniformity (PRNU) noise. The FPN is also used for camera instance identification in [5]. However it can be compensated by subtracting a dark frame from the output image, thus it is not a robust fingerprint and no longer used in later works. The PRNU is directly exploited in $[6,7,8]$. Moreover, PRNU can be also used for camera model identification as proposed in [9] based on the assumption that the fingerprint obtained from images in the TIFF or JPEG format contains traces of post-acquisition processes (e.g. demosaicing) that carry information about the camera model. Other fingerprints include lens aberration [10], Color Filter Array (CFA) pattern and interpolation algorithms [11, 12], and JPEG compression [13], which are proposed for camera model/brand identification.

In general, the image origin identification problem can be formulated into two frameworks: supervised classification [14] and hypothesis testing [15]. Compared with hypothesis testing framework, supervised classification framework is utilized by most of existing methods in the literature to identify camera brands/models. Based on above fingerprint, a forensic feature set is designed and employed in a machine learning algorithm, e.g. Support Vector Machines (SVM) [16]. Supervised classification framework involves three main drawbacks. To achieve high accuracy, this framework requires an expensive training stage that comprises many images with different characteristics (e.g. image content or camera settings) from various sources for representing a real-world situation, which might be unrealistic in practice. Another drawback is the choice of an appropriate forensic feature, which importantly affects the detection performance of the classifier. Besides, the analytic establishment of statistical performances still remains an open problem in supervised classification framework [17].

Even though those methods perform efficiently, they have been designed with a very limited exploitation of hypothesis testing theory and statistical image models. Therefore, their performance can not be analytically established and is only evaluated based on a large image database. Moreover, in the operational context, it is crucial to warrant a prescribed false alarm probability. Our previous work [18] proposed to design a statistical test within hypothesis test-

\footnotetext{
${ }^{1}$ The term source means an individual camera instance, a camera model, or a camera brand.
} 
ing framework based on the heteroscedastic noise model for camera model identification from RAW images. Recently, this work has been extended in [19] for camera model identification from JPEG images based on the generalized noise model [20]. The proposed tests can guarantee a false alarm probability while ensuring a high detection performance on a large database.

\subsection{Main Contributions of the Paper}

This paper aims to design a statistical test for camera model identification from JPEG images, which is based on the same methodology proposed in our previous works $[18,19]$. The approach is based on fingerprint extracted in the Discrete Cosine Transform (DCT) domain. The main motivation behind fingerprint extraction in the DCT domain is that the statistics of DCT coefficients change with different sensor noises combining with various in-camera processing algorithms. Relying on an accurate model of DCT coefficients allows us to capture this statistical difference in natural images taken by different camera models. The main contributions are the following:

- This paper is based on the state-of-the-art statistical model of DCT coefficients [21, 22, 23] for fingerprint extraction. The parameters $(c, d)$ that characterize the linear relation between two parameters $\alpha$ and $\beta$ specified in the proposed model of DCT coefficients are exploited as camera fingerprint for camera model identification.

- Stating the camera model identification problem in hypothesis testing framework, the paper studies the optimal detector given by the Likelihood Ratio Test (LRT) in an ideal context where all model parameters are known. This optimal detector serves as an upper-bound of any statistical test for the camera model identification problem.

- In the practical context, the model parameters are unknown. The paper proposes two Generalized Likelihood Ratio Tests (GLRTs) to deal with the difficulty of unknown parameters. The statistical performance of the GLRTs is analytically established. Moreover, the proposed GLRTs allow the guaranteeing of a prescribed false-alarm rate and the setting of decision threshold independently of the image content, which is crucial in an operational context. Numerical experiments also show that the loss of power of GLRTs compared with the LRT is negligible.

\subsection{Organization of the Paper}

The paper is organized as follows. Section 2 presents the camera fingerprint that is further exploited for camera model identification. Section 3 designs an algorithm for camera fingerprint extraction in the DCT domain. Section 4 states the camera model identification problem in the framework of hypothesis testing theory and studies the LRT assuming that all model parameters are known in advance. Section 5 designs two GLRTs to address the difficulty of unknown parameters. Section 6 presents numerical results of two proposed GLRTs on simulated and real JPEG images. Finally, Section 7 concludes the paper. 


\section{Design of Camera Fingerprint}

To design a camera fingerprint, it is vital to deeply understand image characteristics during various stages of image processing pipeline and study image statistics. Image processing pipeline involves several steps from light capturing to image storage performed in a digital camera, see details about image processing pipeline in [24, 21, 23]. After measuring light intensity at each pixel, RAW image that contains exactly information recorded by the image sensor goes through some typical post-acquisition processes, e.g. demosaicing, white-balancing and gamma correction, to render a full-color high-quality output image, referred to as TIFF image. Image compression can be also performed for ease of storage and transmission.

The study of noise statistics in a natural image from RAW format to TIFF format has been performed in our previous work [20]. Firstly, the approach starts from the heteroscedastic noise model proposed in [18], which is established by modeling various noise sources during RAW image acquisition [25]. The heteroscedastic noise model characterizes more accurately the RAW image than the Additive White Gaussian Noise (AWGN) model widely used in image processing since it takes into account the contribution of Poisson noise in the acquisition process. The heteroscedastic noise model is given as

$$
x_{i} \sim \mathcal{N}\left(\mu_{x_{i}}, a \mu_{x_{i}}+b\right)
$$

where $x_{i}$ denotes a RAW pixel. The index of color channel is omitted for simplicity. By convention, $\mu_{X}$ and $\sigma_{X}^{2}$ denote respectively expectation and variance of a random variable $X$. The parameters $(a, b)$ was proposed in our previous work [18] as fingerprint for camera model identification from RAW images. Then, assuming the operations of demosaicing and white-balancing are linear, that approach [20] takes into account the non-linear effect of gamma correction to develop a generalized noise model, given as

$$
\sigma_{z_{i}}^{2}=\frac{1}{\gamma^{2}} \mu_{z_{i}}^{2-2 \gamma}\left(\tilde{a} \mu_{z_{i}}^{\gamma}+\tilde{b}\right)
$$

where $z_{i}$ denotes an output pixel, $\gamma$ is the correction factor, and $(\tilde{a}, \tilde{b})$ differ from the parameters $(a, b)$ due to the operations of demosaicing and white balancing. It is also shown in [20] that the generalized noise model is relevant to characterize JPEG images with moderate-to-high quality factors ( $Q F \geq 70)$. More details of the generalized noise model are given in [20]. Similarly, the parameters $(\tilde{a}, \tilde{b}, \gamma)$ are proposed in [19] as fingerprint to identify camera models from JPEG images.

The next step in image processing pipeline is JPEG compression that involves transforming the TIFF image into the DCT domain. To capture statistics of DCT coefficients accurately, it is necessary to study the model of DCT coefficients. Based on the assumption that the pixels are identically distributed within $8 \times 8$ block, our previous work $[21,22,23]$ has recently proposed a novel model of DCT coefficients, given by

$$
f_{I}(u)=\sqrt{\frac{2}{\pi}} \frac{\left(|u| \sqrt{\frac{\beta}{2}}\right)^{\alpha-\frac{1}{2}}}{\beta^{\alpha} \Gamma(\alpha)} K_{\alpha-\frac{1}{2}}\left(|u| \sqrt{\frac{2}{\beta}}\right),
$$




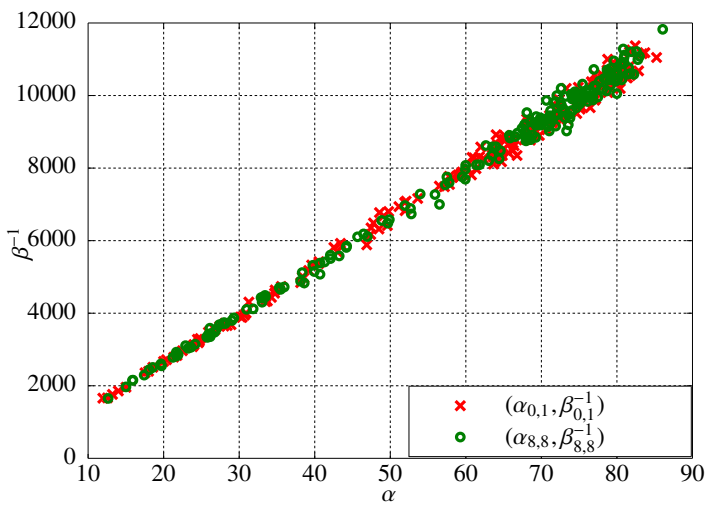

Figure 1: Estimated parameters $(\alpha, \beta)$ at frequency $(0,1)$ and $(8,8)$ of uniform images generated using $\tilde{a}=0.1, \tilde{b}=2, \gamma=2.2$.

where $f_{X}$ denotes the probability density function (pdf) with respect to a random variable $X, \alpha$ is a positive shape parameter, $\beta$ is a positive scale parameter, $\Gamma(\cdot)$ denotes the gamma function and $K_{v}(x)$ denotes the modified Bessel function [26, chap. 5.5]. The proposed model of DCT coefficients outperforms the Laplacian, Generalized Gaussian, and Generalized Gamma model, see more details in [23]. The parameters $(\alpha, \beta)$ can be estimated following the Maximum Likelihood (ML) approach as proposed in [23].

Since the parameters $(\tilde{a}, \tilde{b}, \gamma)$ also contain information about camera model, after transforming into DCT domain, this information is expanded over different frequencies. Therefore, it is proposed to establish the relation between the parameters $(\tilde{a}, \tilde{b}, \gamma)$ and $(\alpha, \beta)$ to capture such information in the DCT domain. For the sake of simplification, this relation is given by

$$
\beta^{-1}=c \alpha+d
$$

where the parameters $(c, d)$ depend on $(\tilde{a}, \tilde{b}, \gamma)$ (see more details in Appendix A). This suggests that the parameters $(c, d)$ can be also used for camera model identification. It can be said that while the relations (1) and (2) characterize the non-stationarity of noise in the spatial domain, the relation (4) characterizes this property in the DCT domain. The relation (4) can capture the difference of noise statistics in natural images taken by different camera models. Moreover, the linearity in relation (4) can facilitate the estimation of the parameters $(c, d)$. It should be noted that in an image whose each $8 \times 8$ block is uniform, the same parameters $(\alpha, \beta)$ and $(c, d)$ are shared among DCT coefficients at different frequencies. The relation (4) on such images is illustrated in Figure 1.

\section{Extraction of Camera Fingerprint}

An important requirement when using the parameters $(c, d)$ as camera fingerprint is that they should be invariant to image content. Furthermore, to guarantee the above mathematical framework, it is necessary to work on homogeneous blocks. These considerations are addressed in this section.

Because of heterogeneity and noise non-stationarity in a natural image, the energy tends to be more located in lower frequencies. Consequently, DCT coefficients at different frequencies do not share the same parameters $(\alpha, \beta)$ 
and $(c, d)$. Therefore, the estimation of parameters $(\alpha, \beta)$ and $(c, d)$ should be performed on each frequency separately. In this paper it is proposed to arrange DCT coefficients into 64 vectors of coefficients according to the zig-zag order. Let $\boldsymbol{I}_{k}=\left(I_{k, 1}, \ldots, I_{k, N}\right)$ with $k \in\{1, \ldots, 64\}$, be the vector of length $N$ that contains coefficients at the frequency $k$. The coefficients $\left(I_{1, i}, \ldots, I_{64, i}\right)$ are in the same block. Analogously, let denote the parameters $\left(\alpha_{k}, \beta_{k}\right)$ and $\left(c_{k}, d_{k}\right)$ with respect to the AC coefficients $\boldsymbol{I}_{k}$.

\subsection{Estimation of Parameters $\left(c_{k}, d_{k}\right)$}

The proposed algorithm for estimation of parameters $\left(c_{k}, d_{k}\right)$ consists of three fundamental steps: image denoising, homogeneous block detection, and Least-Squares (LS) estimation [27]. Image denoising step aims to attenuate the impact of image content. The detection of homogeneous blocks is performed subsequently to provide appropriate sample data for parameter estimation. The LS approach is applied straightforwardly as the relation (4) is linear.

Let $\mathbf{Z}$ be a two-dimensional matrix representing a natural image. To remove the image content, a denoising filter $\mathcal{D}$ is employed so that the residual image $\mathbf{W}$ is given by

$$
\mathbf{W}=\mathbf{Z}-\mathfrak{D}(\mathbf{Z})
$$

If $\mathbf{Z}$ is a color image, the denoising filter $\mathfrak{D}$ is performed on each color component, then three residual components are combined into one residual image using the usual conversion from RGB to grayscale

$$
\mathbf{W}=0.2989 \mathbf{W}_{r}+0.587 \mathbf{W}_{g}+0.114 \mathbf{W}_{b}
$$

where $\mathbf{W}_{r}, \mathbf{W}_{g}$, and $\mathbf{W}_{b}$ are respectively residuals of red, green, and blue component. The residual image $\mathbf{W}$ is then transformed into the DCT domain

$$
\mathbf{I}=\operatorname{DCT}(\mathbf{W})
$$

where $\mathbf{I}$ is the image of DCT coefficients of the residual image $\mathbf{W}$.

For homogeneous block detection, this paper proposes to calculate the standard deviation of each block and compare it with a threshold $\lambda$. The median of absolute deviations (MAD), which is considered as a robust estimator of standard deviation [28], is utilized to calculate the standard deviation of each block. The standard deviation of block $i$ is calculated in the DCT domain as

$$
\hat{s}_{i}=1.4826 \cdot \operatorname{MAD}\left(I_{2, i}, \ldots, I_{64, i}\right) .
$$

The DC coefficient $I_{1, i}$ is excluded in the calculation $\hat{s}_{i}$. The block $i$ is selected if the standard deviation $\hat{s}_{i}$ is smaller than the threshold $\lambda$. The number of selected homogeneous blocks is denoted as $N_{b}$.

Suppose that $L$ couples $\left(\hat{\alpha}_{k, l}, \hat{\beta}_{k, l}\right), l \in\{1, \ldots, L\}$ are available, the LS estimates of the parameters $\left(c_{k}, d_{k}\right)$ are given by

$$
\left(\begin{array}{l}
\hat{c}_{k} \\
\hat{d}_{k}
\end{array}\right)=\left(\mathbf{H}_{k}^{\mathrm{T}} \mathbf{H}_{k}\right)^{-1} \mathbf{H}_{k}^{\mathrm{T}} \boldsymbol{v}_{k}
$$




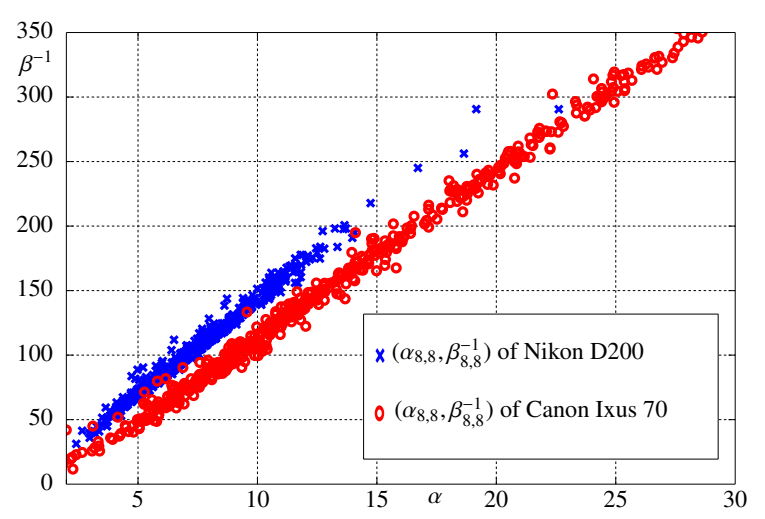

Figure 2: Estimated parameters $(\alpha, \beta)$ at frequency $(8,8)$ of natural JPEG images issued from Canon Ixus 70 and Nikon D200 camera models.

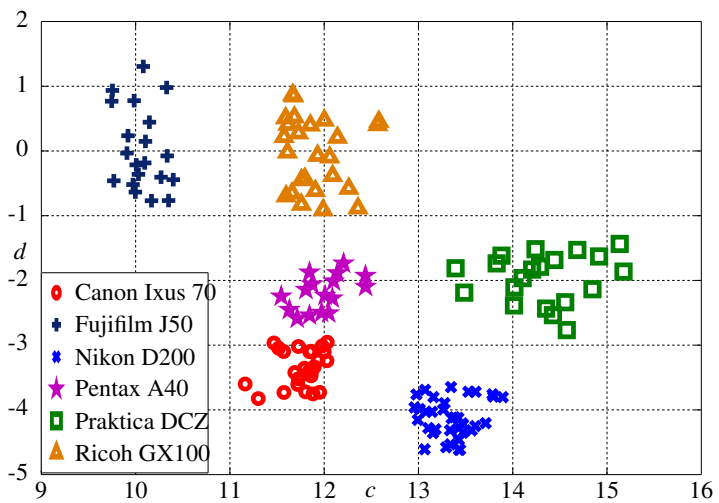

Figure 3: Estimated parameters $(c, d)$ at frequency $(8,8)$ of natural JPEG images issued from different camera models in Dresden database.

with

$$
\mathbf{H}_{k}=\left(\begin{array}{cc}
\hat{\alpha}_{k, 1} & 1 \\
\vdots & \vdots \\
\hat{\alpha}_{k, L} & 1
\end{array}\right) \quad \text { and } \quad \boldsymbol{v}_{k}=\left(\begin{array}{c}
\hat{\beta}_{k, 1}^{-1} \\
\vdots \\
\hat{\beta}_{k, L}^{-1}
\end{array}\right) \text {, }
$$

where $\mathbf{H}_{k}^{\mathrm{T}}$ and $\mathbf{H}_{k}^{-1}$ denote respectively the transpose and inverse of the matrix $\mathbf{H}_{k}$. The LS estimates $\left(\hat{c}_{k}, \hat{d}_{k}\right)$ are unbiased and asymptotically equivalent to ML estimates in large samples [27].

As showed above, the LS approach requires several couples $\left(\alpha_{k}, \beta_{k}\right)$ for estimation of parameters $\left(c_{k}, d_{k}\right)$. One can collect $L$ images and estimate a couple $\left(\alpha_{k, l}, \beta_{k, l}\right)$ from all homogeneous blocks of each image following the ML approach [23]. However, from the practical point of view, it is necessary to estimate the parameters $\left(c_{k}, d_{k}\right)$ from a single image. This is accomplished by extracting randomly a subset of $n_{b}$ homogeneous blocks from $N_{b}$ blocks, then performing the ML estimation of parameters $\left(\alpha_{k, l}, \beta_{k, l}\right)$ on this subset.

\subsection{Property of Parameters $\left(c_{k}, d_{k}\right)$}

When the image content is removed perfectly, the parameters $\left(c_{k}, d_{k}\right)$ remain identical for images with different image contents. However, in practice, due to the fact that the perfect denoising filter $\mathcal{D}$ is difficult to obtain, the DCT 
coefficients at low frequencies are still affected by image content. Meanwhile, the coefficients at high frequencies contain mostly noises because of the energy compaction property of DCT operation. Thus they are more relevant to exploit for camera model identification. Figure 2 shows the the linear relation $(4)$ at frequency $(8,8)$ of natural JPEG images taken by Canon Ixus 70 and Nikon D200 camera models. It should be noted that each point $(\alpha, \beta)$ in Figure 2 corresponds to one image. Figure 2 involves the JPEG images with different imaged scenes, different camera settings, different devices per model, and different environmental conditions. This indicates that the parameters $\left(c_{k}, d_{k}\right)$ remain similar under those conditions.

Moreover, for camera model identification problem, it is necessary to verify the discriminability of parameters $\left(c_{k}, d_{k}\right)$ for different camera models. The Figure 3 shows the parameters $\left(c_{k}, d_{k}\right)$ estimated from JPEG images at frequency $(8,8)$ for different camera models. This figure clearly shows their discriminability between different camera models. Therefore, the parameters $\left(c_{k}, d_{k}\right)$ are proposed to be exploited as camera fingerprint to identify camera models in this paper.

\section{Optimal Detector for Camera Model Identification Problem}

\subsection{Hypothesis Testing Formulation}

Let analyze two camera models $\mathcal{S}_{0}$ and $\mathcal{S}_{1}$. Each camera model $\mathcal{S}_{j}, j \in\{0,1\}$, is characterized by the parameters $\left(c_{k, j}, d_{k, j}\right), k \in\{1, \ldots, K\}$, where $K$ is the number of usable frequencies for camera model identification. For obvious reasons, it is assumed that $\left(c_{k, 0}, d_{k, 0}\right) \neq\left(c_{k, 1}, d_{k, 1}\right)$. In a binary hypothesis testing, the inspected image $\mathbf{Z}$ is either acquired by camera model $\mathcal{S}_{0}$ or camera model $\mathcal{S}_{1}$. The goal of the test is to decide between two hypotheses defined by $\forall k \in\{1, \ldots, K\}, \forall i \in\left\{1, \ldots, N_{b}\right\}$

$$
\left\{\begin{array}{l}
\mathcal{H}_{0}=\left\{I_{k, i} \sim P_{\boldsymbol{\theta}_{k, 0}}, \beta_{k, 0}^{-1}=c_{k, 0} \alpha_{k}+d_{k, 0}\right\} \\
\mathcal{H}_{1}=\left\{I_{k, i} \sim P_{\boldsymbol{\theta}_{k, 1}}, \beta_{k, 1}^{-1}=c_{k, 1} \alpha_{k}+d_{k, 1}\right\},
\end{array}\right.
$$

where $P_{\boldsymbol{\theta}_{k, j}}, \boldsymbol{\theta}_{k, j}=\left(\alpha_{k}, c_{k, j}, d_{k, j}\right)$, denotes the probability distribution of DCT coefficients $I_{k, i}$ under hypothesis $\mathcal{H}_{j}$. As previously explained, this paper focuses on designing a test that allows the guaranteeing of a prescribed false-alarm probability. Hence, let

$$
\mathcal{K}_{\alpha_{0}}=\left\{\delta: \sup _{\boldsymbol{\theta}_{0}} \mathbb{P}_{\mathcal{H}_{0}}\left[\delta(\mathbf{Z})=\mathcal{H}_{1}\right] \leq \alpha_{0}\right\}
$$

be the class of tests whose the false alarm probability is upper-bounded by the prescribed rate $\alpha_{0}$. Here $\boldsymbol{\theta}_{0}=$ $\left(\boldsymbol{\theta}_{1,0}, \ldots, \boldsymbol{\theta}_{K, 0}\right)$ is the vector containing all parameters, $\mathbb{P}_{\mathcal{H}_{j}}[E]$ stands for the probability of event $E$ under hypothesis $\mathcal{H}_{j}, j \in\{0,1\}$, and the supremum over $\boldsymbol{\theta}$ has to be understood as whatever model parameters might be. Among all the tests in the class $\mathcal{K}_{\alpha_{0}}$, it is aimed at finding a test $\delta$ which maximizes the power function, defined by the correct detection probability:

$$
\beta_{\delta}=\mathbb{P}_{\mathcal{H}_{1}}\left[\delta(\mathbf{Z})=\mathcal{H}_{1}\right]
$$


The problem (10) highlights three fundamental difficulties of the camera model identification. First, even when all model parameters $\left(\alpha_{k}, c_{k, j}, d_{k, j}\right)$ are known, the most powerful test, namely the LRT, has never been studied in the literature. The second difficulty concerns unknown parameters $\alpha_{k}$ in practice. Finally, the camera parameters $\left(c_{k, j}, d_{k, j}\right)$ are also unknown, thus the hypothesis $\mathcal{H}_{j}$ becomes composite.

Suppose that the camera model $\mathcal{S}_{0}$ is available, thus forensic analysts can have access to its characteristics, or its fingerprints, i.e. its camera parameters $\left(c_{k, 0}, d_{k, 0}\right)$ can be known. Therefore, they can make a decision by checking whether the image under investigation $\mathbf{Z}$ contains the fingerprint $\left(c_{k, 0}, d_{k, 0}\right)$. It is proposed to solve the problem (10) when the alternative hypothesis $\mathcal{H}_{1}$ is composite, i.e. the camera parameters $\left(c_{k, 1}, d_{k, 1}\right)$ are unknown. It can be noted that a test that maximizes the correct detection probability whatever $\left(c_{k, 1}, d_{k, 1}\right)$ might scarcely exist [15]. The main goal of this paper is to study the LRT and to design the GLRTs to address the second and third difficulties.

\subsection{Likelihood Ratio Test for Two Simple Hypotheses}

When all model parameters are known, in virtue of the Neyman-Pearson lemma [15, theorem 3.2.1], the most powerful test $\delta^{*}$ solving the problem (10) is the LRT given by the following decision rule

$$
\delta^{*}(\mathbf{Z})=\left\{\begin{array}{lll}
\mathcal{H}_{0} & \text { if } & \Lambda(\mathbf{Z})=\sum_{k=1}^{K} \sum_{i=1}^{N_{b}} \Lambda\left(I_{k, i}\right)<\tau^{*} \\
\mathcal{H}_{1} & \text { if } & \Lambda(\mathbf{Z})=\sum_{k=1}^{K} \sum_{i=1}^{N_{b}} \Lambda\left(I_{k, i}\right) \geq \tau^{*}
\end{array}\right.
$$

where the decision threshold $\tau^{*}$ is the solution of the equation

$$
\mathbb{P}_{\mathcal{H}_{0}}\left[\Lambda(\mathbf{Z}) \geq \tau^{*}\right]=\alpha_{0}
$$

to ensure that the LRT is in the class $\mathcal{K}_{\alpha_{0}}$ and the $\operatorname{LR} \Lambda\left(I_{k, i}\right)$ is defined as

$$
\Lambda\left(I_{k, i}\right)=\log \frac{P_{\boldsymbol{\theta}_{k, 1}}\left[I_{k, i}\right]}{P_{\boldsymbol{\theta}_{k, 0}}\left[I_{k, i}\right]},
$$

assuming that the DCT coefficients are statistically independent. From (3), it can be noted that the expression of the $\operatorname{LR} \Lambda\left(I_{k, i}\right)$ is difficult to exploit for subsequent stages, e.g. the design of the GLRT and analytic establishment of its statistical performance. Therefore it is proposed to simplify the $\operatorname{LR} \Lambda\left(I_{k, i}\right)$ to facilitate the study in the manner that it does not cause any loss of optimality.

Using the Laplace's approximation $[29,30]$ (see more details in Appendix B), the function $f_{I}(u)$ can be approximated as

$$
f_{I}(u) \approx \frac{|u|^{\alpha-1}}{(2 \beta)^{\frac{\alpha}{2}} \Gamma(\alpha)} \exp \left(-|u| \sqrt{\frac{2}{\beta}}\right)
$$


Consequently, the $\operatorname{LR} \Lambda\left(I_{k, i}\right)$ can be simplified as

$$
\begin{aligned}
\Lambda\left(I_{k, i}\right) & =\log \frac{\frac{\left|I_{k, i}\right|^{\alpha_{k}-1}}{\left(2 \beta_{k, 1}\right)^{\frac{\alpha_{k}}{2}} \Gamma\left(\alpha_{k}\right)} \exp \left(-\left|I_{k, i}\right| \sqrt{\frac{2}{\beta_{k, 1}}}\right)}{\frac{\left|I_{k, i}\right|^{\alpha_{k}-1}}{\left(2 \beta_{k, 0}\right)^{\frac{\alpha_{k}}{2}} \Gamma\left(\alpha_{k}\right)} \exp \left(-\left|I_{k, i}\right| \sqrt{\frac{2}{\beta_{k, 0}}}\right)} \\
& =\frac{\alpha_{k}}{2} \log \frac{\beta_{k, 1}^{-1}}{\beta_{k, 0}^{-1}}-\sqrt{2}\left|I_{k, i}\right|\left(\sqrt{\beta_{k, 1}^{-1}}-\sqrt{\beta_{k, 0}^{-1}}\right) .
\end{aligned}
$$

It should be noted that other polynomial expansions for the modified Bessel function $K_{v}(x)$ are provided in [26], so a polynomial approximation of $f_{I}(u)$ can be derived. However, those approximations are not considered in this paper. The main advantage of the Laplace's approximation (14) is to provide an approximation of the form of exponential family function, which allows us to simplify the expression of the $\operatorname{LR} \Lambda\left(I_{k, i}\right)$. The approximating function (14) is used only for simplification of the LR. The estimation of parameters $\left(\alpha_{k}, \beta_{k}\right)$ is always based on the exact function (3).

In order to analytically establish the statistical performance of the LRT, it is necessary to characterize the statistical distribution of the LR $\Lambda(\mathbf{Z})$ under each hypothesis $\mathcal{H}_{j}$. To this end, it is proposed to rely on the Lindeberg Central Limit Theorem (CLT) [15, theorem 11.2.5] that requires to calculate the expectation and variance of $\Lambda\left(I_{k, i}\right)$.

Proposition 1. Under hypothesis $\mathcal{H}_{j}$, the first two moments of the $L R \Lambda\left(I_{k, i}\right)$ are given by

$$
\begin{gathered}
m_{k, j} \triangleq \mathbb{E}_{\mathcal{H}_{j}}\left[\Lambda\left(I_{k, i}\right)\right]=\frac{\alpha_{k}}{2} \log \frac{\beta_{k, 1}^{-1}}{\beta_{k, 0}^{-1}} \\
-\frac{2}{\sqrt{\pi}} \beta_{k, j}^{\frac{1}{2}} \frac{\Gamma\left(\alpha_{k}+\frac{1}{2}\right)}{\Gamma\left(\alpha_{k}\right)}\left(\sqrt{\beta_{k, 1}^{-1}}-\sqrt{\beta_{k, 0}^{-1}}\right) \\
v_{k, j} \triangleq \operatorname{Var}_{\mathcal{H}_{j}}\left[\Lambda\left(I_{k, i}\right)\right]=2\left(\sqrt{\beta_{k, 1}^{-1}}-\sqrt{\beta_{k, 0}^{-1}}\right)^{2} \\
\times\left(\alpha_{k} \beta_{k, j}-\frac{2 \beta_{k, j}}{\pi} \frac{\Gamma^{2}\left(\alpha_{k}+\frac{1}{2}\right)}{\Gamma^{2}\left(\alpha_{k}\right)}\right) .
\end{gathered}
$$

where $\mathbb{E}_{\mathcal{H}_{j}}[\cdot]$ and $\operatorname{Var}_{\mathcal{H}_{j}}[\cdot]$ respectively denote the mathematical expectation and variance under hypothesis $\mathcal{H}_{j}$.

Proof. of Proposition 1 is given in Appendix C.

In virtue of Lindeberg CLT, the statistical distribution of the $\operatorname{LR} \Lambda(\mathbf{Z})$ under hypothesis $\mathcal{H}_{j}$ is derived as

$$
\Lambda(\mathbf{Z}) \stackrel{d}{\rightarrow} \mathcal{N}\left(m_{j}, v_{j}\right)
$$

where the notation $\stackrel{d}{\rightarrow}$ denotes the convergence in distribution and the expectation $m_{j}$ and variance $v_{j}$ are given by

$$
\begin{aligned}
m_{j} & =\sum_{k=1}^{K} \sum_{i=1}^{N_{b}} \mathbb{E}_{\mathcal{H}_{j}}\left[\Lambda\left(I_{k, i}\right)\right]=\sum_{k=1}^{K} N_{b} m_{k, j} \\
v_{j} & =\sum_{k=1}^{K} \sum_{i=1}^{N_{b}} \operatorname{Var}_{\mathcal{H}_{j}}\left[\Lambda\left(I_{k, i}\right)\right]=\sum_{k=1}^{K} N_{b} v_{k, j} .
\end{aligned}
$$


Since a natural image is heterogeneous, it is proposed to normalize the LR $\Lambda(Z)$ in order to set the decision threshold independently of the camera parameters. The normalized LR is defined by $\Lambda^{\star}(\mathbf{Z})=\frac{\Lambda(\mathbf{Z})-m_{0}}{\sqrt{v_{0}}}$. Accordingly, the corresponding LRT $\delta^{\star}$ is rewritten as follows

$$
\delta^{\star}(\mathbf{Z})=\left\{\begin{array}{lll}
\mathcal{H}_{0} & \text { if } & \Lambda^{\star}(\mathbf{Z})<\tau^{\star} \\
\mathcal{H}_{1} & \text { if } & \Lambda^{\star}(\mathbf{Z}) \geq \tau^{\star}
\end{array}\right.
$$

where the decision threshold $\tau^{\star}$ is the solution of the equation $\mathbb{P}_{\mathcal{H}_{0}}\left[\Lambda^{\star}(\mathbf{Z}) \geq \tau^{\star}\right]=\alpha_{0}$. The decision threshold $\tau^{\star}$ and the power $\beta_{\delta^{\star}}$ are given in following theorem.

Theorem 1. In an ideal context where all the model parameters $\left(\alpha_{k}, c_{k, j}, d_{k, j}\right)$ are exactly known, the decision threshold and the power function of the LRT $\delta^{\star}$ are given by

$$
\begin{aligned}
\tau^{\star} & =\Phi^{-1}\left(1-\alpha_{0}\right) \\
\beta_{\delta^{\star}} & =1-\Phi\left(\frac{m_{0}-m_{1}+\tau^{\star} \sqrt{v_{0}}}{\sqrt{v_{1}}}\right),
\end{aligned}
$$

where $\Phi(\cdot)$ and $\Phi^{-1}(\cdot)$ denotes respectively the cumulative distribution function of the standard Gaussian random variable and its inverse.

The test power $\beta_{\delta^{\star}}$ serves as an upper-bound of any statistical test for the camera model identification problem. The test $\delta^{\star}$ allows to warrant a prescribed false alarm rate and maximizes the detection probability. Since its statistical performance is analytically established, it can provide an analytically predictable result for any false alarm probability $\alpha_{0}$.

\section{Practical Context: Generalized Likelihood Ratio Test}

The scenario studied in the LRT may not be realistic because the parameters $\left(\alpha_{k}, c_{k, 1}, d_{k, 1}\right)$ are unknown in practice. This section designs two GLRTs to deal with unknown parameters. It is proposed to replace unknown parameters by their ML estimates in the $\operatorname{LR} \Lambda\left(I_{k, i}\right)(15)$.

\subsection{Generalized Likelihood Ratio Test with Unknown Parameters $\alpha_{k}$}

In this subsection it is assumed that the camera parameters $\left(c_{k, j}, d_{k, j}\right)$ are known and we only deal with unknown nuisance parameters $\alpha_{k}$. By replacing unknown parameter $\alpha_{k}$ by its ML estimate $\hat{\alpha}_{k}$ in the LR $\Lambda\left(I_{k, i}\right)(15)$ (see more details about ML estimation of parameters $\left(\alpha_{k}, \beta_{k}\right)$ in [23]), the Generalized Likelihood Ratio (GLR) $\widehat{\Lambda}_{1}\left(I_{k, i}\right)$ can be given by

$$
\begin{aligned}
& \widehat{\Lambda}_{1}\left(I_{k, i}\right)=\frac{\hat{\alpha}_{k}}{2} \log \frac{c_{k, 1} \hat{\alpha}_{k}+d_{k, 1}}{c_{k, 0} \hat{\alpha}_{k}+d_{k, 0}} \\
& -\sqrt{2}\left|I_{k, i}\right|\left(\sqrt{c_{k, 1} \hat{\alpha}_{k}+d_{k, 1}}-\sqrt{c_{k, 0} \hat{\alpha}_{k}+d_{k, 0}}\right)
\end{aligned}
$$


The ML estimate $\hat{\alpha}_{k}$ is asymptotically consistent [15], i.e. it asymptotically converges in probability to its true value: $\hat{\alpha}_{k} \stackrel{p}{\rightarrow} \alpha_{k}$. Therefore, from the Slutsky's theorem [15, theorem 11.2.11], the statistical distribution of the GLR $\widehat{\Lambda}_{1}(\mathbf{Z})=\sum_{k=1}^{K} \sum_{i=1}^{N_{b}} \widehat{\Lambda}_{1}\left(I_{k, i}\right)$ under each hypothesis $\mathcal{H}_{j}$ can be approximated as

$$
\widehat{\Lambda}_{1}(\mathbf{Z}) \stackrel{d}{\rightarrow} \mathcal{N}\left(m_{j}, v_{j}\right)
$$

where the expectation $m_{j}$ and variance $v_{j}$ are given in (19) and (20), respectively.

Similarly, the normalized GLR $\widehat{\Lambda}_{1}^{\star}(\mathbf{Z})$ is defined by $\widehat{\Lambda}_{1}^{\star}(\mathbf{Z})=\frac{\widehat{\Lambda}_{1}(\mathbf{Z})-m_{0}}{\sqrt{v_{0}}}$. However, the expectation $m_{0}$ and variance $v_{0}$ can not be defined in practice since the parameters $\alpha_{k}$ are unknown. Therefore, this paper proposes to replace $\alpha_{k}$ by $\hat{\alpha}_{k}$ in (19) and (20) to obtain the estimates of $m_{0}$ and $v_{0}$, denoted $\hat{m}_{0}$ and $\hat{v}_{0}$. The normalized GLR $\widehat{\Lambda}{ }_{1}^{\star}(\mathbf{Z})$ can be given in practice as $\widehat{\Lambda}_{1}^{\star}(\mathbf{Z})=\frac{\widehat{\Lambda}_{1}(\mathbf{Z})-\hat{m}_{0}}{\sqrt{\hat{v}_{0}}}$. Since the estimates $\hat{m}_{0}$ and $\hat{v}_{0}$ are consistent, it also follows from Slutsky's theorem that

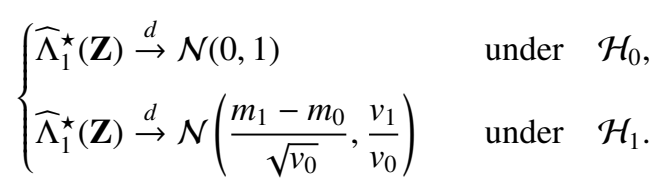

Finally, the GLRT $\widehat{\delta}_{1}^{\star}$ based on the normalized GLR $\widehat{\Lambda}_{1}^{\star}(\mathbf{Z})$ is given by

$$
\widehat{\delta}_{1}^{\star}(\mathbf{Z})=\left\{\begin{array}{lll}
\mathcal{H}_{0} & \text { if } & \widehat{\Lambda}_{1}^{\star}(\mathbf{Z})<\widehat{\tau}_{1}^{\star} \\
\mathcal{H}_{1} & \text { if } & \widehat{\Lambda}_{1}^{\star}(\mathbf{Z}) \geq \widehat{\tau}_{1}^{\star}
\end{array}\right.
$$

where the decision threshold $\widehat{\tau}_{1}^{\star}$ is the solution of the equation $\mathbb{P}_{\mathcal{H}_{0}}\left[\widehat{\Lambda}_{1}^{\star}(\mathbf{Z}) \geq \widehat{\tau}_{1}^{\star}\right]=\alpha_{0}$. From (26), the decision threshold and the power of the GLRT $\widehat{\delta}_{1}^{\star}$ can be accordingly defined as in the Theorem 1 .

\subsection{Generalized Likelihood Ratio Test with Unknown Parameters $\left(\alpha_{k}, c_{k, 1}, d_{k, 1}\right)$}

Before designing the GLRT, the LS estimation of camera parameters $\left(c_{k, 1}, d_{k, 1}\right)$ is performed on the inspected image $\mathbf{Z}$; see Section 3. The LS estimates $\left(\hat{c}_{k, 1}, \hat{d}_{k, 1}\right)$ are asymptotically equivalent to ML estimates in large samples [27]. Moreover, they are unbiaised and follow the asymptotic bivariate Gaussian distribution

$$
\left(\begin{array}{l}
\hat{c}_{k, 1} \\
\hat{d}_{k, 1}
\end{array}\right) \sim \mathcal{N}\left(\left(\begin{array}{l}
c_{k, 1} \\
d_{k, 1}
\end{array}\right),\left(\begin{array}{cc}
\sigma_{c_{k, 1}}^{2} & \sigma_{c_{k, 1} d_{k, 1}} \\
\sigma_{c_{k, 1} d_{k, 1}} & \sigma_{d_{k, 1}}^{2}
\end{array}\right)\right),
$$

where $\sigma_{c_{k, 1}}^{2}, \sigma_{d_{k, 1}}^{2}, \sigma_{c_{k, 1} d_{k, 1}}$ denote the variance of $\hat{c}_{k, 1}$, variance of $\hat{d}_{k, 1}$, and covariance between $\hat{c}_{k, 1}$ and $\hat{d}_{k, 1}$, respectively (see also discussions in Section 6.1 for this covariance matrix). The parameters $\left(c_{k, 1}, d_{k, 1}\right)$ would characterize an unknown camera model. It is require to take into account the variability of LS estimates $\left(\hat{c}_{k, 1}, \hat{d}_{k, 1}\right)$ in the analytic establishment of performance of the GLRT.

By replacing unknown parameters $\left(\alpha_{k}, c_{k, 1}, d_{k, 1}\right)$ by $\left(\hat{\alpha}_{k}, \hat{c}_{k, 1}, \hat{d}_{k, 1}\right)$ in the $\operatorname{LR} \Lambda\left(I_{k, i}\right)(15)$, the $\operatorname{GLR} \widehat{\Lambda}_{2}\left(I_{k, i}\right)$ is given by

$$
\begin{aligned}
& \widehat{\Lambda}_{2}\left(I_{k, i}\right)=\frac{\hat{\alpha}_{k}}{2} \log \frac{\hat{c}_{k, 1} \hat{\alpha}_{k}+\hat{d}_{k, 1}}{c_{k, 0} \hat{\alpha}_{k}+d_{k, 0}} \\
& -\sqrt{2}\left|I_{k, i}\right|\left(\sqrt{\hat{c}_{k, 1} \hat{\alpha}_{k}+\hat{d}_{k, 1}}-\sqrt{c_{k, 0} \hat{\alpha}_{k}+d_{k, 0}}\right) .
\end{aligned}
$$


Proposition 2. Under hypothesis $\mathcal{H}_{j}$, from the Delta method [15, theorem 11.2.14], the first two moments of the GLR $\widehat{\Lambda}_{2}\left(I_{k, i}\right)$ can be approximated as

$$
\begin{aligned}
& \mathbb{E}_{\mathcal{H}_{j}}\left[\widehat{\Lambda}_{2}\left(I_{k, i}\right)\right]=m_{k, j} \\
& \operatorname{Var}_{\mathcal{H}_{j}}\left[\widehat{\Lambda}_{2}\left(I_{k, i}\right)\right]= \\
& v_{k, j}+\frac{\beta_{k, 1}^{2} \alpha_{k}\left(\alpha_{k}+2\right)}{4}\left(\alpha_{k}^{2} \sigma_{c_{k, 1}}^{2}+\sigma_{d_{k, 1}}^{2}+2 \alpha_{k} \sigma_{c_{k, 1} d_{k, 1}}\right) .
\end{aligned}
$$

Proof. of Proposition 2 is given in Appendix D.

For brevity, let denote $\tilde{v}_{k, j}=\operatorname{Var}_{\mathcal{H}_{j}}\left[\widehat{\Lambda}_{2}\left(I_{k, i}\right)\right]$. It can be noted that the second term in (31) aims to take into account the variability of LS estimates $\left(\hat{c}_{k, 1}, \hat{d}_{k, 1}\right)$. In virtue of Lindeberg CLT, the GLR $\widehat{\Lambda}_{2}(\mathbf{Z})=\sum_{k=1}^{K} \sum_{i=1}^{N_{b}} \widehat{\Lambda}_{2}\left(I_{k, i}\right)$ follows the Gaussian distribution under each hypothesis $\mathcal{H}_{j}$

$$
\widehat{\Lambda}_{2}(\mathbf{Z}) \stackrel{d}{\rightarrow} \mathcal{N}\left(m_{j}, \tilde{v}_{j}\right)
$$

where the expectation $m_{j}$ is given in (19) and the variance $\tilde{v}_{j}$ is defined as

$$
\tilde{v}_{j}=\sum_{k=1}^{K} N_{b} \tilde{v}_{k, j}
$$

Finally, the GLRT $\widehat{\delta}_{2}^{\star}$ based on the normalized GLR $\widehat{\Lambda}_{2}^{\star}(\mathbf{Z})=\frac{\widehat{\Lambda}_{2}(\mathbf{Z})-\hat{m}_{0}}{\sqrt{\hat{v}_{0}}}$ is written as

$$
\widehat{\delta}_{2}^{\star}(\mathbf{Z})=\left\{\begin{array}{lll}
\mathcal{H}_{0} & \text { if } & \widehat{\Lambda}_{2}^{\star}(\mathbf{Z})<\widehat{\tau}_{2}^{\star} \\
\mathcal{H}_{1} & \text { if } & \widehat{\Lambda}_{2}^{\star}(\mathbf{Z}) \geq \widehat{\tau}_{2}^{\star}
\end{array}\right.
$$

where the decision threshold $\widehat{\tau}_{2}^{\star}$ is the solution of the equation $\mathbb{P}_{\mathcal{H}_{0}}\left[\widehat{\Lambda}_{2}^{\star}(\mathbf{Z}) \geq \widehat{\tau}_{2}^{\star}\right]=\alpha_{0}$ and $\hat{m}_{0}$ and $\hat{v}_{0}$ are estimates of $m_{0}$ and $\tilde{v}_{0}$ by replacing unknown parameters $\left(\alpha_{k}, c_{k, 1}, d_{k, 1}\right)$ by $\left(\hat{\alpha}_{k}, \hat{c}_{k, 1}, \hat{d}_{k, 1}\right)$ in (19) and (33), respectively. From the Slutsky's theorem [15, theorem 11.2.11], the decision threshold and the power of the GLRT $\widehat{\delta}_{2}^{\star}$ are given in the following theorem.

Theorem 2. When the image $\mathbf{Z}$ is tested against the known camera model $\mathcal{S}_{0}$ characterized by the parameters $\left(c_{k, 0}, d_{k, 0}\right)$, the decision threshold and the power of the GLRT $\widehat{\delta}_{2}^{\star}$ are given by

$$
\begin{aligned}
& \widehat{\tau}_{2}^{\star}=\Phi^{-1}\left(1-\alpha_{0}\right) \\
& \beta_{\tilde{\delta}_{2}^{\star}}=1-\Phi\left(\frac{m_{0}-m_{1}+\widehat{\tau}_{2}^{\star} \sqrt{\tilde{v}_{0}}}{\sqrt{\tilde{v}_{1}}}\right) .
\end{aligned}
$$

The statistical performance of the proposed GLRTs $\widehat{\delta}_{1}^{\star}$ and $\widehat{\delta}_{2}^{\star}$ is analytically provided. Moreover, they allow us to warrant a prescribed false alarm rate and set the decision threshold independently of camera parameters (see (22) and (35)). It is worth noting that the GLRT dealing with unknown parameters $\alpha_{k}$ while the camera parameters $\left(c_{k, j}, d_{k, j}\right)$ are known can be interpreted as a closed hypothesis testing since the decision is made only between two known 


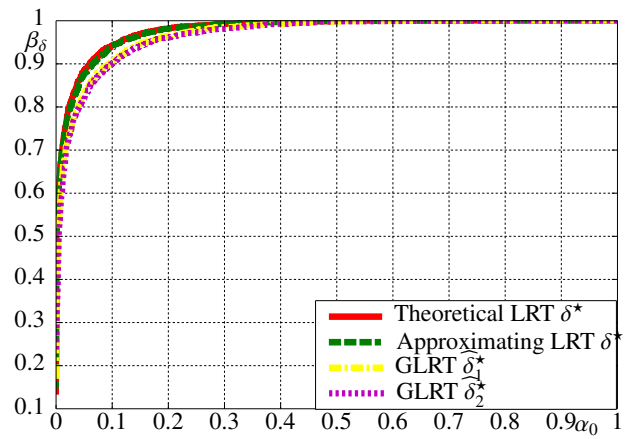

Figure 4: Detection performance of proposed tests on simulated vectors with 1024 coefficients.

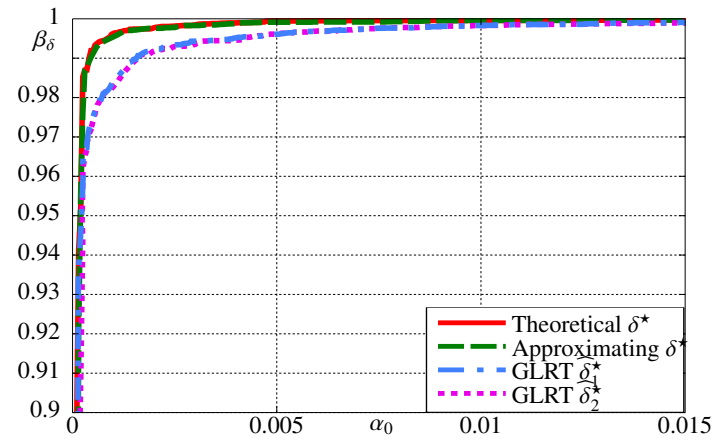

Figure 5: Detection performance of proposed tests on simulated vectors with 4096 coefficients.

camera models $\mathcal{S}_{0}$ and $\mathcal{S}_{1}$. Meanwhile, the GLRT dealing with unknown camera parameters $\left(c_{k, 1}, d_{k, 1}\right)$ becomes an open hypothesis testing telling whether the given image is acquired by camera model $\mathcal{S}_{0}$ or not. The given image is allowed to be acquired by an unknown camera model. Therefore, two proposed tests can be straightforwardly applied, depending on the requirements of the operational context.

\section{Numerical Experiments}

In this paper, the wavelet-based denoising filter proposed in $[31,4]$ is employed to suppress image content because of its relative accuracy and computational efficiency. Besides, the selection of homogeneous blocks requires an appropriate threshold $\lambda$. This threshold should be fixed independently of image content. The threshold $\lambda$ is set at $\lambda=0.5$.

\subsection{Detection Performance on Simulated Database}

The implementation of the GLRT $\widehat{\delta}_{2}^{\star}$ requires to know the covariance matrix of LS estimates $\left(\hat{c}_{k, 1}, \hat{d}_{k, 1}\right)$. However, the ML estimates $\left(\hat{\alpha}_{k}, \hat{\beta}_{k}\right)$ are solved numerically, which causes a difficulty of defining their statistical properties. Thus it seems impossible to establish the covariance matrix of $\left(\hat{c}_{k}, \hat{d}_{k}\right)$ analytically. To overcome this difficulty, it is proposed to estimate the parameters $\left(c_{k}, d_{k}\right)$ on each image from 50 images taken by the camera model $\mathcal{S}_{0}$ since this camera 


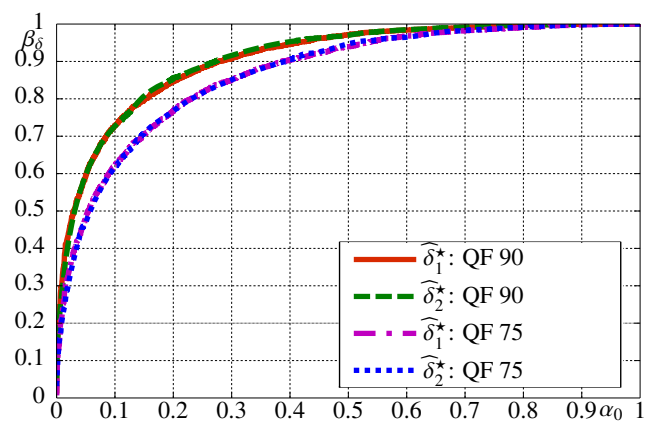

Figure 6: Detection performance of proposed tests for 1024 coefficients at frequency $(8,8)$ extracted randomly from simulated images with different quality factors.

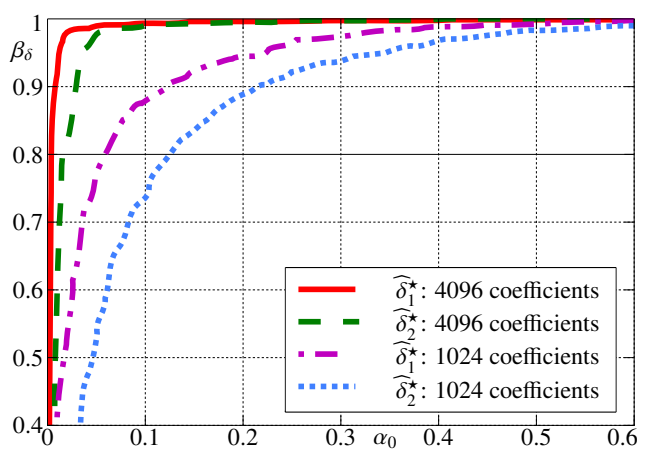

Figure 7: Detection performance of proposed tests for different number of coefficients at frequency $(8,8)$ of natural JPEG images taken by Canon Ixus 70 and Nikon D200 camera models.

model is assumed to be available. Then the empirical covariance matrix can be calculated from previous couples $\left(\hat{c}_{k}, \hat{d}_{k}\right)$. Speaking rigorously, this is the covariance matrix characterizing the variability of the camera parameters $\left(c_{k, 0}, d_{k, 0}\right)$. By doing so, it is expected that the parameters $\left(\hat{c}_{k, 1}, \hat{d}_{k, 1}\right)$ fall into the neighborhood of $\left(c_{k, 0}, d_{k, 0}\right)$, namely that the inspected image $\mathbf{Z}$ is acquired by the camera model $\mathcal{S}_{0}$. This leads us to exploit this covariance matrix in the implementation of the GLRT $\widehat{\delta}_{2}^{\star}$. This step is also performed in the test with real images.

The detection performance of proposed tests is first theoretically studied on simulated database. Suppose that the camera models $\mathcal{S}_{0}$ and $\mathcal{S}_{1}$ are characterized by the parameters $\left(c_{0}, d_{0}\right)=(11.8,-3.5)$ and $\left(c_{1}, d_{1}\right)=(13.5,-4.5)$, respectively. These parameters correspond to frequency $(8,8)$ of JPEG images taken by Canon Ixus 70 and Nikon D200 camera models in the Dresden image database [32], respectively (see Figure 2). They are used to generate randomly 5000 vectors of 1024 and 4096 coefficients under $\mathcal{H}_{0}$ and $\mathcal{H}_{1}$. Because this paper proposes to simplify the $\operatorname{LR} \Lambda\left(I_{k, i}\right)$ to facilitate the study, it is desirable to compare the detection performance of the LRT based on the approximating LR with the one based on the exact LR. The expectation and variance of the exact LR are calculated numerically. Moreover, it is necessary to compare the detection performance of the proposed GLRTs with the LRT since the GLRTs utilize ML estimates of unknown parameters, which may cause a loss of power. Figure 4 and Figure 5 show the detection performance of all proposed tests for 1024 and 4096 coefficients, respectively. For clarity, only 


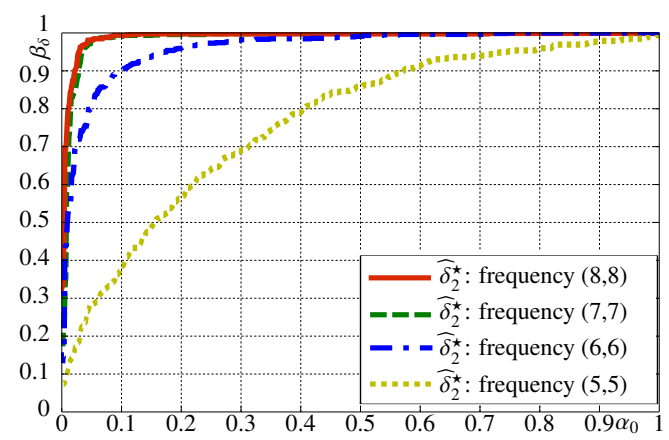

Figure 8: Detection performance of the GLRT $\widehat{\delta}_{2}^{\star}$ for 4096 coefficients at different frequencies of natural JPEG images taken by Canon Ixus 70 and Nikon D200 camera models.

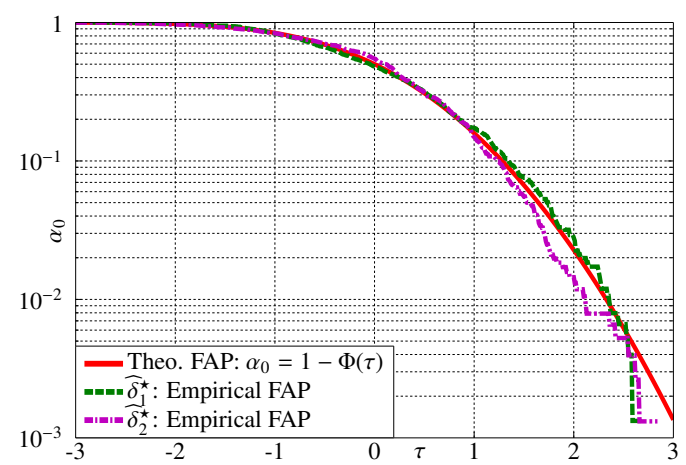

Figure 9: Comparison between the theoretical false alarm probability (FAP) and the empirical FAP, plotted as a function of decision threshold $\tau$.

regions of interest are illustrated in the figures. It is worth noting that the loss of power between the theoretical LRT and approximating LRT is negligible. Besides, a small loss of power is revealed between the GLRTs and LRT due to the estimation of unknown parameters. Nevertheless this loss of power decreases when the number of coefficients increases. It can be also noted that the loss of power between two GLRTs $\widehat{\delta}_{1}^{\star}$ and $\widehat{\delta}_{2}^{\star}$ is negligible, i.e. the variability of estimates $\left(\hat{c}_{k, 1}, \hat{d}_{k, 1}\right)$ are well taken into account in the GLRT $\widehat{\delta}_{2}^{\star}$. The power function of all proposed tests is perfect (e.g. $\beta_{\delta}=1$ ) from $2^{14}$ coefficients for any false alarm rate $\alpha_{0}$.

Moreover, it is desirable to study the detection performance of the proposed tests on simulated images that follow the image processing pipeline as described in Section 2. To this end, suppose the camera models $\mathcal{S}_{0}$ and $\mathcal{S}_{1}$ are characterized by the parameters $\left(\tilde{a}_{0}, \tilde{b}_{0}, \gamma_{0}\right)=(0.1,2,2.2)$ and $\left(\tilde{a}_{1}, \tilde{b}_{1}, \gamma_{1}\right)=(0.2,2,2.2)$. These parameters are used together with the reference image lena to generate randomly 5000 images under $\mathcal{H}_{0}$ and $\mathcal{H}_{1}$. The simulated images are then compressed with quality factor of 90 and 75. The detection performance of the proposed GLRTs for 1024 coefficients at frequency $(8,8)$ extracted randomly from those simulated images is shown in Figure 6. As expected, a small loss of power is revealed with the decline of quality factor. 


\begin{tabular}{lc|ccc}
\hline \hline Camera Model & & No. devices & Resolution & No. images \\
\hline Canon Ixus 70 & $\mathrm{C}$ & 3 & $3072 \times 2304$ & 350 \\
Fujifilm FinePix J50 & $\mathrm{F}$ & 3 & $3264 \times 2448$ & 350 \\
Kodak M1063 & $\mathrm{K}$ & 5 & $3664 \times 2748$ & 550 \\
Nikon Coolpix S710 & $\mathrm{N} 1$ & 5 & $4352 \times 3264$ & 550 \\
Nikon D200 & $\mathrm{N} 2$ & 2 & $3872 \times 2592$ & 250 \\
Nikon D70 & $\mathrm{N} 3$ & 2 & $3008 \times 2000$ & 250 \\
Pentax Optio A40 & $\mathrm{Pe}$ & 4 & $4000 \times 3000$ & 450 \\
Praktica DCZ 5.9 & $\mathrm{Pr}$ & 5 & $2560 \times 1920$ & 550 \\
Ricoh Capilo GX100 & $\mathrm{Ri}$ & 5 & $3648 \times 2736$ & 550 \\
Rollei RCP-7325XS & $\mathrm{Ro}$ & 3 & $3072 \times 2304$ & 350 \\
Sony DSC-H50 & $\mathrm{S}$ & 2 & $3456 \times 2592$ & 250 \\
\hline$\sum$ & 11 & 39 & & 4450 \\
\hline \hline
\end{tabular}

Table 1: Camera Model Used in Experiments

\subsection{Detection Performance on Two Canon Ixus 70 and Nikon D200 Camera Models}

It is important to remind that the proposed GLRTs are designed in the framework of hypothesis testing theory where the reference camera parameters $\left(c_{k, 0}, d_{k, 0}\right)$ under hypothesis $\mathcal{H}_{0}$ are assumed to be known in advance. Therefore, those parameters need to be defined accurately in practice. To this end, the parameters $\left(c_{k}, d_{k}\right)$ are estimated on 50 images of the camera model $\mathcal{S}_{0}$ and the reference parameter $\left(c_{k, 0}, d_{k, 0}\right)$ is calculated as the average of previous estimates $\left(\hat{c}_{k}, \hat{d}_{k}\right)$. Evidently, using more images will get a better estimate but it is also less realistic. The number of 50 is a good trade-off.

To highlight the relevance of the proposed GLRTs, two Canon Ixus 70 and Nikon D200 camera models of the Dresden image database [32] are chosen to conduct experiments. The Canon Ixus 70 and Nikon D200 cameras are respectively set at $\mathcal{H}_{0}$ and $\mathcal{H}_{1}$. All available JPEG images of each camera model are used in this experiment. The reference camera parameters are estimated as discussed above. The Figure 7 shows the detection performance of the GLRTs $\widehat{\delta}_{1}^{\star}$ and $\widehat{\delta}_{2}^{\star}$ for 1024 and 4096 coefficients extracted randomly at frequency $(8,8)$ of natural JPEG images taken by Canon Ixus 70 and Nikon D200 camera models. We can note a similar behavior to the detection performance on simulated database. Besides, there is a small loss of power between the two GLRTs because different estimates $\left(\hat{c}_{k, 1}, \hat{d}_{k, 1}\right)$ used in the design of the GLRT $\widehat{\delta}_{2}^{\star}$ are still influenced by image content. Nevertheless, this loss of power also decreases when the number of coefficients increases. Besides, Figure 8 illustrates detection performance of the GLRT $\widehat{\delta}_{2}^{\star}$ for 4096 coefficients randomly extracted at different frequencies. It can be noted that the detection performance decreases with the reverse zig-zag order.

Meanwhile, the Figure 9 shows the comparison between the theoretical and empirical false alarm probability, which are plotted as a function of decision threshold $\tau$. The two proposed GLRTs $\widehat{\delta}_{1}^{\star}$ and $\widehat{\delta}_{2}^{\star}$ show an ability of guaranteeing a prescribed false alarm rate, even though there is a slight difference in some cases (typically $\alpha_{0} \leq 10^{-3}$ ) 


\begin{tabular}{|c|c|c|c|c|c|c|c|c|c|c|c|c|c|}
\hline & & \multicolumn{11}{|c|}{ Tested images } & \multirow[b]{2}{*}{ Avg. } \\
\hline & & $\mathrm{Cn} 2$ & N1 & $\mathrm{Pe}$ & F & Ro & $\mathrm{K}$ & N3 & N2 & $\operatorname{Pr}$ & $\mathrm{Ri}$ & $S$ & \\
\hline & $\mathrm{Cn} 2$ & 100 & 26.54 & 20.42 & $*$ & $*$ & $*$ & $*$ & $*$ & $*$ & $*$ & $*$ & \\
\hline & N1 & * & 84.61 & 10.56 & $*$ & $*$ & $*$ & $*$ & $*$ & $*$ & $*$ & $*$ & \\
\hline & $\mathrm{Pe}$ & $*$ & 7.79 & 82.04 & * & $*$ & $*$ & $*$ & $*$ & $*$ & $*$ & $*$ & \\
\hline & $\mathrm{F}$ & $*$ & $*$ & * & 91.67 & 6.63 & $*$ & $*$ & $*$ & $*$ & $*$ & $*$ & \\
\hline & Ro & $*$ & $*$ & $*$ & 22.92 & 100 & $*$ & $*$ & $*$ & * & $*$ & $*$ & \\
\hline \multirow[t]{7}{*}{$\mathcal{H}_{0}$} & $\mathrm{~K}$ & $*$ & $*$ & $*$ & $*$ & $*$ & 99.81 & 14.15 & $*$ & $*$ & $*$ & $*$ & \\
\hline & $\mathrm{N} 3$ & $*$ & $*$ & $*$ & $*$ & $*$ & $*$ & 97.62 & $*$ & $*$ & $*$ & $*$ & \\
\hline & $\mathrm{N} 2$ & $*$ & $*$ & $*$ & $*$ & $*$ & $*$ & $*$ & 100 & $*$ & $*$ & $*$ & \\
\hline & $\operatorname{Pr}$ & $*$ & $*$ & $*$ & * & $*$ & $*$ & $*$ & $*$ & 95.78 & $*$ & $*$ & \\
\hline & $\mathrm{Ri}$ & $*$ & $*$ & $*$ & $*$ & $*$ & $*$ & $*$ & $*$ & $*$ & 100 & $*$ & \\
\hline & \multirow[t]{2}{*}{$S$} & $*$ & $*$ & * & $*$ & $*$ & $*$ & $*$ & $*$ & $*$ & $*$ & 96.81 & \\
\hline & & & & & & & & & & & & & 95.31 \\
\hline
\end{tabular}

Table 2: Detection performance of proposed detector $\widehat{\delta}_{2}^{\star}$ (the symbol * represents values smaller than $2 \%$ )

due to the influence of image content and the inaccuracy of the CLT for modeling tails.

\subsection{Detection Performance on a Large Image Database}

Experiments are then conducted on a large database to verify the efficiency of the proposed approach. The public Dresden image database [32] is chosen in our experiments. Technical specifications of the cameras are shown in Table 1 , see more details in [32]. The database covers different devices per camera model, different imaged scenes, different camera settings and different environmental conditions. All images are acquired with the highest available JPEG quality setting and maximum available resolution. For each camera model, the set contains 50 images per camera model for estimation of reference camera parameters and 100 images per device for testing, which are randomly selected from the Dresden database.

Firstly, the GLRT $\widehat{\delta}_{2}^{\star}$ is used to verify whether a given image is acquired by the camera model of interest. The decision threshold $\widehat{\tau}_{2}^{\star}$ is given by the Theorem 2 corresponding to the false alarm rate $\alpha_{0}=10^{-5}$. If the normalized GLR $\widehat{\Lambda}_{2}^{\star}(\mathbf{Z})$ is smaller than the decision threshold $\widehat{\tau}_{2}^{\star}$, the hypothesis $\mathcal{H}_{0}$ is accepted, i.e. the given image is taken from the camera model of interest. On the contrary, the hypothesis $\mathcal{H}_{1}$ is accepted. It is proposed to use the last 21 high frequencies for the test. The detection performance of the test $\widehat{\delta}_{2}^{\star}$ is shown in Table 2. In this table, each camera model is considered as hypothesis $\mathcal{H}_{0}$ (row) and all images (column) are tested against $\mathcal{H}_{0}$. The values in the table indicate the percentage of images that are detected taken by the camera model $\mathcal{H}_{0}$. The table in this paper is not used in the same way as in the classification in which the sum for each class yields $100 \%$. The inspected image is brought into the binary testing of the known camera model $\mathcal{H}_{0}$ against the others, thus the sum of a class may not yield $100 \%$. It could lead to a scenario that an image is declared taken by at least two camera models. It can be noted from Table 2 that the 


\begin{tabular}{|c|c|c|c|c|c|c|c|c|c|c|c|c|c|}
\hline & & \multicolumn{11}{|c|}{ Tested images } & \multirow[b]{2}{*}{ Avg. } \\
\hline & & $\mathrm{Cn} 2$ & N1 & $\mathrm{Pe}$ & $\mathrm{F}$ & Ro & $\mathrm{K}$ & N3 & N2 & $\operatorname{Pr}$ & $\mathrm{Ri}$ & $S$ & \\
\hline & $\mathrm{Cn} 2$ & 98.26 & $*$ & $*$ & $*$ & $*$ & $*$ & $*$ & 2.61 & $*$ & $*$ & $*$ & \\
\hline & N1 & $*$ & 100 & 3.97 & $*$ & $*$ & $*$ & $*$ & $*$ & $*$ & $*$ & $*$ & \\
\hline & $\mathrm{Pe}$ & $*$ & $*$ & 90.86 & $*$ & $*$ & $*$ & $*$ & $*$ & $*$ & $*$ & $*$ & \\
\hline & $\mathrm{F}$ & $*$ & $*$ & $*$ & 99.94 & $*$ & $*$ & $*$ & $*$ & $*$ & $*$ & $*$ & \\
\hline & Ro & $*$ & $*$ & $*$ & $*$ & 99.79 & $*$ & $*$ & $*$ & $*$ & $*$ & $*$ & \\
\hline \multirow[t]{7}{*}{$\mathcal{H}_{0}$} & $\mathrm{~K}$ & $*$ & $*$ & $*$ & $*$ & $*$ & 99.19 & $*$ & $*$ & $*$ & $*$ & $*$ & \\
\hline & $\mathrm{N} 3$ & * & $*$ & $*$ & $*$ & $*$ & $*$ & 84.57 & $*$ & $*$ & $*$ & $*$ & \\
\hline & N2 & $*$ & $*$ & $*$ & $*$ & $*$ & $*$ & $*$ & 93.73 & $*$ & $*$ & $*$ & \\
\hline & $\operatorname{Pr}$ & $*$ & $*$ & $*$ & $*$ & $*$ & $*$ & $*$ & $*$ & 98.42 & $*$ & $*$ & \\
\hline & $\mathrm{Ri}$ & $*$ & $*$ & $*$ & $*$ & $*$ & $*$ & $*$ & $*$ & $*$ & 100 & $*$ & \\
\hline & \multirow[t]{2}{*}{$S$} & $*$ & $*$ & $*$ & $*$ & $*$ & $*$ & $*$ & $*$ & $*$ & $*$ & 98.23 & \\
\hline & & & & & & & & & & & & & 96.63 \\
\hline
\end{tabular}

Table 3: Detection performance of SVM-based detector [32]

incorrect detection in some groups of camera models, such as (C,N1,Pe), (F,Ro), and (K,N3), is important. This may be justified due to a similarity in JPEG compression scheme used in the camera. To deal with this scenario, a second testing round involves performing the GLRT $\widehat{\delta}_{1}^{\star}$ on the camera models of conflict. The detection performance of the test $\widehat{\delta}_{1}^{\star}$ is shown in Table 5. The images are almost correctly classified. It should be noted that the GLRT $\widehat{\delta}_{1}^{\star}$ aims to give a decision rule between two different known camera models, thus the experiment on the diagonal of Table 5 is not performed. This paper also presents the SVM-based detector for comparison, which has been already performed in [32] on the Dresden database using 46 different features to capture characteristics of different camera components of a digital camera. The detection performance of this SVM-based detector is shown in Table 3. The proposed detector $\widehat{\delta}_{2}^{\star}$ is slightly equivalent to the SVM-based detector in terms of average correct detection performance but the misclassification of the former is more severe. The PRNU-based detector [7] is also performed in this experiment. This PRNU-based detector is only conducted on one device per model. Its detection performance is shown in Table 4. Overall, the two proposed detectors provide an equivalent detection performance compared with the other ones in the literature, but the latter can not allow to guarantee a prescribed false alarm probability like the proposed detectors.

Remark 1. The present paper proposes to exploit the state-of-the-ar model of DCT coefficients provided in [23]. This model is not only more accurate than prior-art models in the literature, but also is the only one that is mathematically justified based on a statistical analysis of images' properties according to the image processing pipeline, as provided in [23]. Therefore, relying on the proposed model allows us to capture accurately statistics of DCT coefficients as well as to analyze camera fingerprint that can be exploited for camera model identification. 


\begin{tabular}{|c|c|c|c|c|c|c|c|c|c|c|c|c|c|}
\hline & & \multicolumn{11}{|c|}{ Tested images } & \multirow[b]{2}{*}{ Avg. } \\
\hline & & $\mathrm{Cn} 2$ & N1 & $\mathrm{Pe}$ & F & Ro & $\mathrm{K}$ & $\mathrm{N} 3$ & $\mathrm{~N} 2$ & $\operatorname{Pr}$ & $\mathrm{Ri}$ & S & \\
\hline \multirow{12}{*}{$\mathcal{H}_{0}$} & $\mathrm{Cn} 2$ & 100 & $*$ & $*$ & $*$ & $*$ & $*$ & $*$ & $*$ & $*$ & $*$ & $*$ & \\
\hline & $\mathrm{N} 1$ & $*$ & 98.92 & $*$ & $*$ & $*$ & $*$ & $*$ & $*$ & $*$ & $*$ & $*$ & \\
\hline & $\mathrm{Pe}$ & $*$ & $*$ & 100 & $*$ & $*$ & $*$ & $*$ & $*$ & $*$ & $*$ & $*$ & \\
\hline & F & $*$ & $*$ & * & 73.81 & $*$ & * & * & * & * & * & $*$ & \\
\hline & Ro & $*$ & $*$ & $*$ & $*$ & 99.49 & $*$ & $*$ & $*$ & $*$ & $*$ & $*$ & \\
\hline & K & $*$ & * & * & * & * & 99.19 & * & $*$ & * & $*$ & $*$ & \\
\hline & N3 & $*$ & $*$ & * & $*$ & * & $*$ & 100 & $*$ & $*$ & $*$ & $*$ & \\
\hline & N2 & $*$ & $*$ & $*$ & $*$ & $*$ & $*$ & $*$ & 98.92 & * & $*$ & * & \\
\hline & $\operatorname{Pr}$ & $*$ & * & * & $*$ & $*$ & $*$ & $*$ & $*$ & 96.65 & $*$ & $*$ & \\
\hline & $\mathrm{Ri}$ & $*$ & * & $*$ & $*$ & $*$ & $*$ & $*$ & $*$ & * & 100 & $*$ & \\
\hline & \multirow[t]{2}{*}{ S } & $*$ & $*$ & $*$ & $*$ & $*$ & $*$ & $*$ & $*$ & $*$ & $*$ & 100 & \\
\hline & & & & & & & & & & & & & 96.99 \\
\hline
\end{tabular}

Table 4: Detection performance of PRNU-based detector [7]

\begin{tabular}{cc|ccccccc}
\hline \hline & & \multicolumn{7}{|c}{$\mathcal{H}_{1}$} \\
& & $\mathrm{Cn} 2$ & $\mathrm{~N} 1$ & $\mathrm{Pe}$ & $\mathrm{F}$ & $\mathrm{Ro}$ & $\mathrm{K}$ & $\mathrm{N} 3$ \\
\hline \multirow{4}{*}{$\mathcal{H}_{0}$} & $\mathrm{Cn} 2$ & - & 0 & 3.52 & - & - & - & - \\
& $\mathrm{N} 1$ & 0 & - & 3.13 & - & - & - & - \\
& $\mathrm{Pe}$ & 0 & 0 & - & - & - & - & - \\
& $\mathrm{F}$ & - & - & - & - & 0 & - & - \\
& $\mathrm{Ro}$ & - & - & - & 2.12 & - & - & - \\
& $\mathrm{K}$ & - & - & - & - & - & - & 0 \\
& $\mathrm{~N} 3$ & - & - & - & - & - & 0 & - \\
\hline \hline
\end{tabular}

Table 5: Detection performance of proposed detector $\widehat{\delta}_{1}^{\star}$

\section{Conclusion}

The goal of this paper is to design a statistical test for camera model identification from JPEG images within hypothesis testing framework. The approach is based on the state-of-the-art model of DCT coefficients to capture their statistical difference, which jointly results from different sensor noises and in-camera processing algorithms. The parameters $(c, d)$ characterizing the simplistic linear relation between $\alpha$ and $\beta^{-1}$, which are two parameters of the DCT coefficient model, are proposed to be exploited as camera fingerprint for camera model identification. Based on the parametric model of DCT coefficients, this paper studies the most powerful LRT and proposes two GLRTs that can be straightforwardly applied in practice. The strength of the proposed approach is that statistical performance of the tests can be analytically established as well as they can warrant a prescribed false alarm rate while ensuring a high detection performance. 


\section{Acknowledgements}

The authors would like to thank the Digital Data Embedding Laboratory, University of Binghamton, especially J. Fridrich, for the use of the High Performance Computing server, allowing a considerable reduction in execution time of our algorithm on the large image database.

\section{Appendix A. Relation between the Parameters $(\tilde{a}, \tilde{b}, \gamma)$ and $\left(\alpha_{p, q}, \beta_{p, q}\right)$}

Given an arbitrary image $\mathbf{Z}$, the DCT operation is performed in each $8 \times 8$ block of $\mathbf{Z}$ as follows

$$
\begin{aligned}
I_{p, q}= & \frac{1}{4} T_{p} T_{q} \sum_{m=0}^{7} \sum_{n=0}^{7} z_{m, n} \\
& \times \cos \left(\frac{(2 m+1) p \pi}{16}\right) \cos \left(\frac{(2 n+1) q \pi}{16}\right),
\end{aligned}
$$

where $z_{m, n}$ denotes a pixel within a $8 \times 8$ block of $\mathbf{Z}, 0 \leq m \leq 7,0 \leq n \leq 7$ and $I_{p, q}$ denotes the two-dimensional DCT coefficient and

$$
T_{p}= \begin{cases}\frac{1}{\sqrt{2}} & \text { for } p=0 \\ 1 & \text { for } p>0 .\end{cases}
$$

For the sake of simplification, it is assumed that the pixels are independent and identically distributed within each $8 \times 8$ block. By taking variance on the both sides of the equation (A.1), it follows that

$$
\begin{aligned}
\operatorname{Var}\left[I_{p, q}\right] & =\operatorname{Var}\left[z_{m, n}\right] \\
\operatorname{Var}\left[I_{p, q}^{2}\right] & =S_{p, q} \operatorname{Var}\left[z_{m, n}^{2}\right]+\left(1-S_{p, q}\right) \operatorname{Var}^{2}\left[z_{m, n}\right],
\end{aligned}
$$

with

$$
\begin{aligned}
S_{p, q}= & \frac{1}{4^{4}} T_{p}^{4} T_{q}^{4} \sum_{m=0}^{7} \sum_{n=0}^{7} \\
& \cos ^{4}\left(\frac{(2 m+1) p \pi}{16}\right) \cos ^{4}\left(\frac{(2 n+1) q \pi}{16}\right) .
\end{aligned}
$$

In fact, the proposed model of DCT coefficients (3) is based on the doubly stochastic model given in [33] that takes into account the statistics of DCT coefficients in a block whose variance is constant and the variability of block variance in a natural image. Given a constant block variance $\sigma_{b}^{2}$, the AC coefficient $I$ may be approximately distributed as zero-mean Gaussian in virtue of the CLT. Besides, the block variance $\sigma_{b}^{2}$ can be approximately modeled by the Gamma distribution $\mathcal{G}(\alpha, \beta)$. As the pdf of $I$ is symmetric, the odd moments vanish. Based on the law of total 
expectation, the short calculation shows that

$$
\begin{aligned}
\operatorname{Var}\left[I_{p, q}\right] & =\mathbb{E}_{I_{p, q}}\left[I_{p, q}^{2}\right]=\mathbb{E}_{\sigma_{b}^{2}}\left[\mathbb{E}_{I_{p, q} \mid \sigma_{b}^{2}}\left[I_{p, q}^{2} \mid \sigma_{b}^{2}\right]\right]=\mathbb{E}_{\sigma_{b}^{2}}\left[\sigma_{b}^{2}\right] \\
& =\alpha_{p, q} \beta_{p, q} \\
\operatorname{Var}\left[I_{p, q}^{2}\right] & =\mathbb{E}\left[I_{p, q}^{4}\right]-\mathbb{E}^{2}\left[I_{p, q}^{2}\right]=\mathbb{E}_{\sigma_{b}^{2}}\left[3 \sigma_{b}^{4}\right]-\alpha_{p, q}^{2} \beta_{p, q}^{2} \\
& =2 \alpha_{p, q}^{2} \beta_{p, q}^{2}+3 \alpha_{p, q} \beta_{p, q}^{2} .
\end{aligned}
$$

On the other hand, it follows from [20] that

$$
\begin{aligned}
& \operatorname{Var}\left[z_{m, n}\right]=\frac{1}{\gamma^{2}} \mu_{z_{m, n}}^{2-2 \gamma}\left(\tilde{a} \mu_{z_{m, n}}^{\gamma}+\tilde{b}\right) \\
& \operatorname{Var}\left[z_{m, n}^{2}\right]=\frac{4}{\gamma^{2}} \mu_{z_{m, n}}^{4-2 \gamma}\left(\tilde{a} \mu_{z_{m, n}}^{\gamma}+\tilde{b}\right) .
\end{aligned}
$$

Consequently, one derives that

$$
\begin{aligned}
\alpha_{p, q} \beta_{p, q} & =\frac{1}{\gamma^{2}} \mu_{z_{m, n}}^{2-2 \gamma}\left(\tilde{a} \mu_{z_{m, n}}^{\gamma}+\tilde{b}\right) \\
\left(S_{p, q}+1\right) \alpha_{p, q} \beta_{p, q}+3 \beta_{p, q} & =4 S_{p, q} \mu_{z_{m, n}}^{2} .
\end{aligned}
$$

Resolving this system of equations, the relation between $\alpha_{p, q}$ and $\beta_{p, q}^{-1}$ is given as

$$
\begin{aligned}
\beta_{p, q}^{-1} & =\frac{\left(S_{p, q}+1\right) \alpha_{p, q}+3}{4 \tilde{b}^{\frac{2}{\gamma}} S_{p, q}} \\
& \times\left(\sqrt{\gamma^{2} \alpha_{p, q} \tilde{b} \frac{\left(S_{p, q}+1\right) \alpha_{p, q}+3}{4 S_{p, q}}+\frac{\tilde{a}^{2}}{4}}-\frac{\tilde{a}}{2}\right)^{\frac{2}{\gamma}} .
\end{aligned}
$$

This relation is too complicated to exploit. Therefore, it is proposed to employ the polynomial expansion and only keep the first two terms

$$
\beta_{p, q}^{-1}=c_{p, q} \alpha_{p, q}+d_{p, q}
$$

where the parameters $\left(c_{p, q}, d_{p, q}\right)$ depend on the parameters $(\tilde{a}, \tilde{b}, \gamma)$. Numerical experiments show that this simplified equation sufficiently characterizes the relation between the parameters $(\tilde{a}, \tilde{b}, \gamma)$ and $\left(\alpha_{p, q}, \beta_{p, q}\right)$ (see Figure 1 and Figure 2).

\section{Appendix B. Laplace's Approximation of DCT Coefficient Model}

Let us briefly describe the idea behind the Laplace's approximation [29]. The Laplace's method aims to provide an approximation for integrals of the form

$$
I=\int \exp (-g(t)) d t
$$

when the function $g(t)$ reaches the global minimum at $t^{*}$. By using the Taylor expansion of the function $g(t)$ at $t^{*}$, we have

$$
g(t)=g\left(t^{*}\right)+\frac{g^{\prime \prime}\left(t^{*}\right)}{2}\left(t-t^{*}\right)^{2}+o\left(\left(t-t^{*}\right)^{2}\right),
$$


where $g^{\prime \prime}(t)$ denotes the second derivative of the function $g(t)$. Therefore, the integral $\mathcal{I}$ can be approximated as

$$
\mathcal{I} \approx \exp \left(-g\left(t^{*}\right)\right) \int \exp \left[-\frac{g^{\prime \prime}\left(t^{*}\right)}{2}\left(t-t^{*}\right)^{2}\right] d t .
$$

This integral takes the form of Gaussian integral. We derive

$$
\mathcal{I} \approx \sqrt{\frac{2 \pi}{\left|g^{\prime \prime}\left(t^{*}\right)\right|}} \exp \left(-g\left(t^{*}\right)\right) .
$$

A generalization has been made in [30] with an arbitrary function $h(t)$

$$
\mathcal{I}=\int h(t) \exp (-g(t)) d t \approx \sqrt{\frac{2 \pi}{\left|g^{\prime \prime}\left(t^{*}\right)\right|}} h\left(t^{*}\right) \exp \left(-g\left(t^{*}\right)\right) .
$$

As described in [23], the DCT coefficient model $f_{I}(u)$ is rewritten as follows

$$
f_{I}(u)=\frac{1}{\sqrt{2 \pi} \beta^{\alpha} \Gamma(\alpha)} \int_{0}^{\infty} h(t) \exp \left(-g\left(t^{*}\right)\right) d t
$$

where

$$
g(t)=\frac{t}{\beta}+\frac{u^{2}}{2 t} \quad \text { and } \quad h(t)=t^{\alpha-\frac{3}{2}}
$$

The function $g(t)$ reaches the minimum at $t^{*}=|u| \sqrt{\frac{\beta}{2}}$ and its second derivative is defined by $g^{\prime \prime}(t)=\frac{u^{2}}{t^{3}}$. Consequently, the function $f_{I}(u)$ can be approximated as

$$
f_{I}(u) \approx \frac{|u|^{\alpha-1}}{(2 \beta)^{\frac{\alpha}{2}} \Gamma(\alpha)} \exp \left(-|u| \sqrt{\frac{2}{\beta}}\right) .
$$

It can be noted that this approximating model is a special case of the GГ model [34] when $\gamma=1$ (the variable $\gamma$ is given in [34, Eq. (6)]).

\section{Appendix C. Expectation and Variance of the $\operatorname{LR} \Lambda\left(I_{k, i}\right)$ under Hypothesis $\mathcal{H}_{j}$}

It can be noted from (15) that it is necessary to calculate the expectation and variance of the random variable $|I|$. Given a constant variance $\sigma_{b}^{2}$, the random variable $I$ is normally distributed with zero-mean and variance $\sigma_{b}^{2}$. Thus, the random variable $|I|$ follows the half-Normal distribution [35]. Therefore, we obtain

$$
\mathbb{E}_{I \mid \sigma_{b}^{2}}\left[|I| \mid \sigma_{b}^{2}\right]=\sqrt{\frac{2}{\pi}} \sigma_{b}
$$

Based on the law of total expectation, the mathematical expectation of $|I|$ is given by

$$
\begin{aligned}
\mathbb{E}_{I}[|I|] & =\mathbb{E}_{\sigma_{b}^{2}}\left[\mathbb{E}_{I \mid \sigma_{b}^{2}}\left[|I| \mid \sigma_{b}^{2}\right]\right]=\sqrt{\frac{2}{\pi}} \mathbb{E}_{\sigma_{b}^{2}}\left[\sigma_{b}\right] \\
& =\sqrt{\frac{2}{\pi}} \beta^{\frac{1}{2}} \frac{\Gamma\left(\alpha+\frac{1}{2}\right)}{\Gamma(\alpha)} .
\end{aligned}
$$


Besides, the variance of $|I|$ is given by

$$
\begin{aligned}
\operatorname{Var}_{I}[|I|] & =\mathbb{E}_{I}\left[|I|^{2}\right]-\mathbb{E}_{I}^{2}[|I|] \\
& =\alpha \beta-\frac{2 \beta}{\pi} \frac{\Gamma^{2}\left(\alpha+\frac{1}{2}\right)}{\Gamma^{2}(\alpha)} .
\end{aligned}
$$

Consequently, the expectation and variance of the $\operatorname{LR} \Lambda\left(I_{k, i}\right)$ under hypothesis $\mathcal{H}_{j}$ can be defined by

$$
\begin{aligned}
& \mathbb{E}_{\mathcal{H}_{j}}\left[\Lambda\left(I_{k, i}\right)\right]=\frac{\alpha_{k}}{2} \log \frac{\beta_{k, 1}^{-1}}{\beta_{k, 0}^{-1}} \\
&-\frac{2}{\sqrt{\pi}} \beta_{k, j}^{\frac{1}{2}} \frac{\Gamma\left(\alpha_{k}+\frac{1}{2}\right)}{\Gamma\left(\alpha_{k}\right)}\left(\sqrt{\beta_{k, 1}^{-1}}-\sqrt{\beta_{k, 0}^{-1}}\right) \\
& \operatorname{Var}_{\mathcal{H}_{j}}\left[\Lambda\left(I_{k, i}\right)\right]= 2\left(\sqrt{\beta_{k, 1}^{-1}}-\sqrt{\beta_{k, 0}^{-1}}\right)^{2} \\
& \times\left(\alpha_{k} \beta_{k, j}-\frac{2 \beta_{k, j}}{\pi} \frac{\Gamma^{2}\left(\alpha_{k}+\frac{1}{2}\right)}{\Gamma^{2}\left(\alpha_{k}\right)}\right) .
\end{aligned}
$$

\section{Appendix D. Asymptotic Expectation and Variance of the GLR $\widehat{\Lambda}_{2}\left(I_{k, i}\right)$ under Hypothesis $\mathcal{H}_{j}$}

It is assumed that the variance of the estimate $\hat{\alpha}_{k}$ is negligible when the number of coefficients $N_{b}$ is very large. Thus it is proposed to treat the estimate $\hat{\alpha}_{k}$ as a constant $\alpha_{k}$. Besides, since the estimates $\left(\hat{c}_{k, 1}, \hat{d}_{k, 1}\right)$ are consistent, the asymptotic mathematical expectation of the GLR $\widehat{\Lambda}_{2}\left(I_{k, i}\right)$ under hypothesis $\mathcal{H}_{j}$ does not change, i.e. $\mathbb{E}_{\mathcal{H}_{j}}\left[\widehat{\Lambda}_{2}\left(I_{k, i}\right)\right]=$ $m_{k, j}$.

Meanwhile, the variance of the GLR $\widehat{\Lambda}_{2}\left(I_{k, i}\right)$ needs to take into account the variability of the estimates $\left(\hat{c}_{k, 1}, \hat{d}_{k, 1}\right)$. Based on the definitions of mathematical expectation and variance, we have

$$
\begin{aligned}
\mathbb{E}_{\mathcal{H}_{j}}\left[\hat{c}_{k, 1} \hat{\alpha}_{k}+\hat{d}_{k, 1}\right] & =\beta_{k, 1}^{-1} \\
\operatorname{Var}_{\mathcal{H}_{j}}\left[\hat{c}_{k, 1} \hat{\alpha}_{k}+\hat{d}_{k, 1}\right] & =\alpha_{k}^{2} \sigma_{c_{k, 1}}^{2}+\sigma_{d_{k, 1}}^{2}+2 \alpha_{k} \sigma_{c_{k, 1}} d_{k, 1}
\end{aligned}
$$

Subsequently, from the Delta method [15, theorem 11.2.14], we derive that

$$
\begin{aligned}
\operatorname{Var}_{\mathcal{H}_{j}} & {\left[\log \frac{\hat{c}_{k, 1} \hat{\alpha}_{k}+\hat{d}_{k, 1}}{c_{k, 0} \hat{\alpha}_{k}+d_{k, 0}}\right]=\operatorname{Var}_{\mathcal{H}_{j}}\left[\log \left(\hat{c}_{k, 1} \hat{\alpha}_{k}+\hat{d}_{k, 1}\right)\right] } \\
= & \frac{\operatorname{Var}_{\mathcal{H}_{j}}\left[\hat{c}_{k, 1} \hat{\alpha}_{k}+\hat{d}_{k, 1}\right]}{\mathbb{E}_{\mathcal{H}_{j}}^{2}\left[\hat{c}_{k, 1} \hat{\alpha}_{k}+\hat{d}_{k, 1}\right]} \\
= & \beta_{k, 1}^{2}\left(\alpha_{k}^{2} \sigma_{c_{k, 1}}^{2}+\sigma_{d_{k, 1}}^{2}+2 \alpha_{k} \sigma_{c_{k, 1} d_{k, 1}}\right),
\end{aligned}
$$

and

$$
\begin{aligned}
& \operatorname{Var}_{\mathcal{H}_{j}}\left[\sqrt{\hat{c}_{k, 1} \hat{\alpha}_{k}+\hat{d}_{k, 1}}-\sqrt{c_{k, 0} \hat{\alpha}_{k}+d_{k, 0}}\right] \\
& =\operatorname{Var}_{\mathcal{H}_{j}}\left[\sqrt{\hat{c}_{k, 1} \hat{\alpha}_{k}+\hat{d}_{k, 1}}\right]=\frac{\operatorname{Var}_{\mathcal{H}_{j}}\left[\hat{c}_{k, 1} \hat{\alpha}_{k}+\hat{d}_{k, 1}\right]}{4 \mathbb{E}_{\mathcal{H}_{j}}\left[\hat{c}_{k, 1} \hat{\alpha}_{k}+\hat{d}_{k, 1}\right]} \\
& =\frac{\beta_{k, 1}}{4}\left(\alpha_{k}^{2} \sigma_{c_{k, 1}}^{2}+\sigma_{d_{k, 1}}^{2}+2 \alpha_{k} \sigma_{c_{k, 1} d_{k, 1}}\right) .
\end{aligned}
$$


Finally, the asymptotic variance of the GLR $\widehat{\Lambda}_{2}\left(I_{k, i}\right)$ can be given as

$$
\begin{aligned}
& \operatorname{Var}_{\mathcal{H}_{j}}\left[\widehat{\Lambda}_{2}\left(I_{k, i}\right)\right]=\frac{\alpha_{k}^{2}}{4} \operatorname{Var}_{\mathcal{H}_{j}}\left[\log \frac{\hat{c}_{k, 1} \hat{\alpha}_{k}+\hat{d}_{k, 1}}{c_{k, 0} \hat{\alpha}_{k}+d_{k, 0}}\right] \\
& +2 \operatorname{Var}_{\mathcal{H}_{j}}\left[\sqrt{\hat{c}_{k, 1} \hat{\alpha}_{k}+\hat{d}_{k, 1}}-\sqrt{c_{k, 0} \hat{\alpha}_{k}+d_{k, 0}}\right] \mathbb{E}_{\mathcal{H}_{j}}\left[|I|^{2}\right] \\
& +2 \mathbb{E}_{\mathcal{H}_{j}}^{2}\left[\sqrt{\hat{c}_{k, 1} \hat{\alpha}_{k}+\hat{d}_{k, 1}}-\sqrt{c_{k, 0} \hat{\alpha}_{k}+d_{k, 0}}\right] \operatorname{Var}_{\mathcal{H}_{j}}\left[|I|^{2}\right] \\
& =\frac{\beta_{k, 1}^{2} \alpha_{k}\left(\alpha_{k}+2\right)}{4}\left(\alpha_{k}^{2} \sigma_{c_{k, 1}}^{2}+\sigma_{d_{k, 1}}^{2}+2 \alpha_{k} \sigma_{c_{k, 1} d_{k, 1}}\right) \\
& +2\left(\sqrt{\beta_{k, 1}^{-1}}-\sqrt{\beta_{k, 0}^{-1}}\right)^{2}\left(\alpha_{k} \beta_{k, j}-\frac{2 \beta_{k, j}}{\pi} \frac{\Gamma^{2}\left(\alpha_{k}+\frac{1}{2}\right)}{\Gamma^{2}\left(\alpha_{k}\right)}\right) .
\end{aligned}
$$

\section{References}

[1] M. C. Stamm, M. Wu, K. J. R. Liu, Information forensics: An overview of the first decade, IEEE Access 1 (2013) 167-200.

[2] C. I. Podilchuk, E. J. Delp, Digital watermarking: algorithms and applications, IEEE Signal Process. Mag. 18 (4) (2001) $33-46$.

[3] G. L. Friedman, The trustworthy digital camera: restoring credibility to the photographic image, IEEE Trans. Consum. Electron. 39 (4) (1993) 905-910.

[4] J. Lukas, J. Fridrich, M. Goljan, Digital camera identification from sensor pattern noise, IEEE Trans. Inf. Forensics Security 1 (2) (2006) $205-214$.

[5] K. Kurosawa, K. Kuroki, N. Saitoh, CCD fingerprint method-identification of a video camera from videotaped images, in: Proc. IEEE Int. Conf. Image Process., Vol. 3, 1999, pp. 537-540.

[6] M. Chen, J. Fridrich, M. Goljan, J. Lukas, Determining image origin and integrity using sensor noise, IEEE Trans. Inf. Forensics Security 3 (1) (2008) 74-90.

[7] J. Fridrich, Digital image forensics, IEEE Signal Process. Mag. 26 (2) (2009) 26-37.

[8] C.-T. Li, Y. Li, Color-decoupled photo response non-uniformity for digital image forensics, IEEE Trans. Circuits Syst. Video Technol. 22 (2) (2012) 260-271.

[9] T. Filler, J. Fridrich, M. Goljan, Using sensor pattern noise for camera model identification, in: Proc. IEEE Int. Conf. Image Process., 2008, pp. 1296-1299.

[10] K. S. Choi, E. Y. Lam, K. Wong, Source camera identification using footprints from lens aberration, in: Proc. SPIE Digit. Photography, Vol. 6069,2006 , pp. 172-179.

[11] A. Swaminathan, M. Wu, K. J. R. Liu, Nonintrusive component forensics of visual sensors using output images, IEEE Trans. Inf. Forensics Security 2 (1) (2007) 91-106.

[12] H. Cao, A. C. Kot, Accurate detection of demosaicing regularity for digital image forensics, IEEE Trans. Inf. Forensics Security 4 (4) (2009) 899-910.

[13] K. S. Choi, E. Y. Lam, K. K. Y. Wong, Source camera identification by JPEG compression statistics for image forensics, in: IEEE Region Conf. TENCON, 2006, pp. 1-4.

[14] T. Mitchell, Machine Learning, McGraw Hill, 1997.

[15] E. L. Lehmann, J. P. Romano, Testing Statistical Hypotheses, 3rd Edition, Springer, New york, 2005.

[16] V. N. Vapnik, The Nature of Statistical Learning Theory, 2nd Edition, Springer Verlag, 2000.

[17] C. Scott, Performance measures for Neyman-Pearson classification, IEEE Trans. Inf. Theory 53 (8) (2007) $2852-2863$.

[18] T. H. Thai, R. Cogranne, F. Retraint, Camera model identification based on the heteroscedastic noise model, IEEE Trans. Image Process. 23 (1) (2014) 250-263.

[19] T. H. Thai, F. Retraint, R. Cogranne, Camera model identification based on the generalized noise model in natural images, Signal Processing (under review). 
[20] T. H. Thai, F. Retraint, R. Cogranne, Generalized signal-dependent noise model and parameter estimation for natural images, Signal Processing (under review).

[21] T. H. Thai, R. Cogranne, F. Retraint, Statistical model of natural images, in: Proc. IEEE. Int. Conf. Image Process., 2012, pp. $2525-2528$.

[22] T. H. Thai, R. Cogranne, F. Retraint, Steganalysis of jsteg algorithm based on a novel statistical model of quantized DCT coefficients, in: Proc. IEEE Int. Conf. Image Process., 2013, pp. 4427-4431.

[23] T. H. Thai, R. Cogranne, F. Retraint, Statistical model of quantized DCT coefficients: Application in the steganalysis of Jsteg algorithm, IEEE Trans. Image Process. 23 (5) (2014) 1980-1993.

[24] R. Ramanath, W. E. Snyder, Y. Yoo, M. S. Drew, Color image processing pipeline, IEEE Signal Process. Mag. 22 (1) (2005) $34-43$.

[25] G. E. Healey, R. Kondepudy, Radiometric CCD camera calibration and noise estimation, IEEE Trans. Pattern Anal. Mach. Intell. 16 (3) (1994) 267-276.

[26] I. M. Ryzhik, I. S. Gradshteyn, Tables of Integrals, Series, and Products, Elsevier, United Kingdom, 2007.

[27] C. R. Rao, H. Toutenburg, Linear models : Least Squares and Alternatives, 2nd Edition, Springer, New york, 1999.

[28] P. J. Rousseeuw, C. Croux, Alternatives to the median absolute deviation, J. Amer. Statist. Assoc. 88 (424) (1993) $1273-1283$.

[29] L. Tierney, J. B. Kadane, Accurate approximations for posterior moments and marginal densities, J. Amer. Statist. Assoc. 81 (393) (1986) 82-86.

[30] R. Butler, A. Wood, Laplace approximations for hypergeometric functions with matrix argument, Ann. Statist. 30 (4) (2002) $1155-1177$.

[31] M. K. Mihçak, I. Kozintsev, K. Ramchandran, Spatially adaptive statistical modeling of wavelet image coefficients and its application to denoising, in: Proc. IEEE Int. Conf. Acoust., Speech, Signal Process., Vol. 6, 1999, pp. 3253-3256.

[32] T. Gloe, R. Bohme, The Dresden image database for benchmarking digital image forensics, in: Proc. ACM Symposium Applied Comput., Vol. 2, 2010, pp. 1585-1591.

[33] E. Y. Lam, J. W. Goodman, A mathematical analysis of the DCT coefficient distributions for images, IEEE Trans. Image Process. 9 (10) (2000) 1661-1666.

[34] J.-H. Chang, J.-W. Shin, N. S. Kim, S. K. Mitra, Image probability distribution based on generalized gamma function, IEEE Signal Process. Lett., 12 (4) (2005) 325-328.

[35] N. L. Johnson, S. Kotz, N. Balakrishnan, Continuous univariate distributions, 2nd Edition, Wiley, New york, 1994. 